\title{
Connexin 43 confers chemoresistance through activating PI3K
}

\author{
Kevin J. Pridham (D) ${ }^{1}$, Farah Shah ${ }^{1,2}$, Kasen R. Hutchings $\mathbb{D}^{1,2}{ }^{1,}$ Kevin L. Sheng ${ }^{3}$, Sujuan Guo ${ }^{1}$, Min Liu ${ }^{1}$, Pratik Kanabur ${ }^{1,2}$, \\ Samy Lamouille ${ }^{1,4,5}$, Gabrielle Lewis ${ }^{1}$, Marc Morales ${ }^{1}$, Jane Jourdan ${ }^{1}$, Christina L. Grek ${ }^{6}$, Gautam G. Ghatnekar ${ }^{6}$, Robin Varghese ${ }^{3}$, \\ Deborah F. Kelly ${ }^{7,8,9}$, Robert G. Gourdie (iD ${ }^{1,10,11 \bowtie}$ and Zhi Sheng $\mathbb{D}^{1,2,11 \bowtie}$
}

(c) The Author(s) 2022

Circumventing chemoresistance is crucial for effectively treating cancer including glioblastoma, a lethal brain cancer. The gap junction protein connexin 43 (Cx43) renders glioblastoma resistant to chemotherapy; however, targeting Cx43 is difficult because mechanisms underlying Cx43-mediated chemoresistance remain elusive. Here we report that Cx43, but not other connexins, is highly expressed in a subpopulation of glioblastoma and Cx43 mRNA levels strongly correlate with poor prognosis and chemoresistance in this population, making Cx43 the prime therapeutic target among all connexins. Depleting Cx43 or treating cells with aCT1-a Cx43 peptide inhibitor that sensitizes glioblastoma to the chemotherapy temozolomide-inactivates phosphatidylinositol-3 kinase (PI3K), whereas overexpression of Cx43 activates this signaling. Moreover, aCT1-induced chemosensitization is counteracted by a PI3K active mutant. Further research reveals that aCT1 inactivates PI3K without blocking the release of PI3K-activating molecules from membrane channels and that $C x 43$ selectively binds to the PI3K catalytic subunit $\beta$ (PIK3CB, also called PI3K $\beta$ or $\mathrm{p} 110 \beta$ ), suggesting that $\mathrm{C} \times 43$ activates $\mathrm{PIK} 3 \mathrm{CB} / \mathrm{p} 110 \beta$ independent of its channel functions. To explore the therapeutic potential of simultaneously targeting Cx43 and PIK3CB/p110 $\beta$, aCT1 is combined with TGX-221 or GSK2636771, two $\mathrm{PIK} 3 \mathrm{CB} / \mathrm{p} 110 \beta$-selective inhibitors. These two different treatments synergistically inactivate PI3K and sensitize glioblastoma cells to temozolomide in vitro and in vivo. Our study has revealed novel mechanistic insights into Cx43/PI3K-mediated temozolomide resistance in glioblastoma and demonstrated that targeting Cx43 and PIK3CB/p110 $\beta$ together is an effective therapeutic approach for overcoming chemoresistance.

Oncogenesis (2022)11:2 ; https://doi.org/10.1038/s41389-022-00378-7

\section{INTRODUCTION}

Overcoming resistance to chemotherapy such as temozolomide (TMZ) has proven perplexing and remains a key unmet clinical need. As an alkylating agent, TMZ reacts with DNA at multiple sites, yielding $\mathrm{O}^{6}$-methylguanine lesions that subsequently induce DNA breaks and eventually cell death [1]. Given that TMZ is able to pass the blood-brain barrier [2], this drug has been used as the frontline chemotherapy for glioblastoma (GBM) an aggressive and lethal cancer that accounts for approximately half of all malignant brain tumors and has a grim prognosis with an average survival time of 14.6 months $[3,4]$. Adding to this dismal outcome, nearly $90 \%$ of patients with GBM succumb to tumor recurrence and the average survival for recurrent GBM is about 5.5-7.5 months due to limited therapeutic options and resistance to TMZ [5]. Hence, overcoming $T M Z$ resistance is key to effectively treating GBM and curbing GBM progression. Poor responses of nearly $50 \%$ of GBM patients to TMZ are due to the expression of 0-6-methylguanine-DNA methyltransferase (MGMT) [6, 7]. MGMT repairs TMZ-induced DNA damage, conferring MGMT-dependent TMZ resistance; as such, inhibiting
MGMT has shown encouraging clinical benefits [8]. Patients with no MGMT expression also develop MGMT-independent resistance to TMZ $[9,10]$. Factors involved in MGMT-independent TMZ resistance include the DNA mismatch repair pathway and genetic alterations $[11,12]$. However, targeting these factors to circumvent TMZ resistance has been a daunting task. Deeper insights into MGMTindependent $T M Z$ resistance are therefore needed.

Recently, several lines of evidence have indicated that the gap junction protein connexin 43 (Cx43; also known as gap junction protein A1, GJA1), a channel-forming protein important for intercellular communication [13], controls the response of GBM cells to TMZ. Ectopic expression of Cx43 renders GBM cells resistant to TMZ [14-17], and blocking Cx43 using different approaches such as antibodies or channel inhibitors restores TMZ sensitivity [14-20]. However, it remains unclear whether Cx43mediated TMZ resistance depends on MGMT. Our recent work [21] reveals that high levels of Cx43 in MGMT-deficient GBM cell lines and primary patient samples correlate with poor responses to TMZ and that aCT1, a clinically-tested therapeutic peptide that

\footnotetext{
${ }^{1}$ Fralin Biomedical Research Institute at VTC, Roanoke, VA 24016, USA. ${ }^{2}$ Department of Internal Medicine, Virginia Tech Carilion School of Medicine, Roanoke, VA 24016, USA. ${ }^{3}$ Department of Biomedical Affairs and Research, Edward Via College of Osteopathic Medicine, Blacksburg, VA 24060, USA. ${ }^{4}$ Department of Basic Science Education, Virginia Tech Carilion School of Medicine, Roanoke, VA 24016, USA. ${ }^{5}$ Department of Biological Sciences, Virginia Tech, Blacksburg, VA 24061, USA. ${ }^{6}$ FirstString Research, Inc, Mount Pleasant, SC 29464, USA. ${ }^{7}$ Department of Biomedical Engineering, Pennsylvania State University, University Park, PA 16802, USA. ${ }^{8}$ Huck Institutes of the Life Sciences, Pennsylvania State

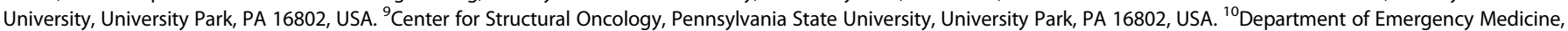
Virginia Tech Carilion School of Medicine, Roanoke, VA 24016, USA. ${ }^{11}$ Faculty of Health Science, Virginia Tech, Blacksburg, VA 24061, USA. ${ }^{\circledR}$ email: gourdier@vtc.vt.edu; zhisheng@vtc.vt.edu
}

Received: 22 June 2021 Revised: 8 December 2021 Accepted: 30 December 2021

Published online: 12 January 2022 
comprises the Cx43 carboxyl terminus (CT) and an antennapedia cell-penetrating sequence [22], antagonizes TMZ resistance. Nonetheless, the molecular underpinnings of Cx43-mediated $\mathrm{TMZ}$ resistance remain elusive, making it difficult to effectively target $\mathrm{Cx} 43$ to treat GBM.

In this report, we determined the role of connexins in GBM prognosis and TMZ resistance, explored how Cx43 activates phosphatidylinositol-3 kinase (PI3K) independent of $\mathrm{C} \times 43$ channels and induces TMZ resistance, and examined a candidate triple combinational therapy entailing the $\mathrm{Cx} 43$ inhibitor aCT1, PI3Kselective inhibitors, and TMZ in preclinical studies for its effectiveness in overcoming TMZ resistance.

\section{MATERIALS AND METHODS \\ Reagents}

Resources and catalog numbers of reagents were included in Supplemental Table S1. Chemical compounds were reconstituted in dimethyl sulfoxide (DMSO) at a concentration of $50-80 \mathrm{mM}$. Peptide aCT1 and Gap27 were in vitro synthesized and purchased from LifeTein, LLC. Lyophilized peptide was reconstituted in 1x PBS $(137 \mathrm{mM} \mathrm{NaCl}, 2.7 \mathrm{mM} \mathrm{KCl}$, $10 \mathrm{mM} \mathrm{Na}_{2} \mathrm{HPO}_{4}$, and $1.8 \mathrm{mM} \mathrm{KH}_{2} \mathrm{PO}_{4}$ ) at a concentration of 5 or $10 \mathrm{mM}$. All chemicals were aliquoted and stored at $-80^{\circ} \mathrm{C}$.

\section{Cell culture}

GBM cell lines, primary GBM cells, GBM stem cells (GSCs), and human astrocytes were cultured as previously described [21, 23-30]. Cell lines have been authenticated by the ATCC authentication service utilizing Short Tandem Repeat profiling and tested for mycoplasma contamination. Primary cells VTC-001, VTC-003, VTC-005, and VTC-103 were cultured in DMEM supplemented with $15 \%$ fetal bovine serum (Peak Serum, Inc.) and penicillin/streptomycin. Normal human astrocytes were cultured in MCDB131 medium (Sigma) containing 3\% fetal bovine serum (Peak Serum, Inc.), 10x G-5 Supplement (Gibco), and penicillin/streptomycin. Primary GBM cells were kept at low passages (no more than 10).

\section{Analysis of online databases}

Analysis of online databases has been described previously [21, 26-29]. Transcriptomic and proteomic datasets with corresponding clinical information and immunostaining data of human tissues are downloaded from the following websites: (1) The Cancer Gene Atlas (TCGA) datasets: https://www.cbioportal.org and https://gliovis.shinyapps.io/GlioVis/; (2) Gravendeel, Rembrandt, Lee Y, and Murat GBM: https://gliovis.shinyapps. io/GlioVis/; (3) The China Glioma Gene Atlas (CGGA) datasets: https:// gliovis.shinyapps.io/GlioVis/; (4) GBM cell lines from the Cancer Dependency Map (DepMap): https://depmap.org/portal/; (5) The Human Protein Atlas: https://www.proteinatlas.org. The Kaplan-Meier survival analysis or the Cox hazard proportional model were used to determine the association of gene expression and patient survival. The Pearson correlation coefficient, calculated using Prism 9 software, was used to determine the expression correlation between different genes/proteins, The histologic images from The Human Protein Atlas were quantified using Image J and further analyzed using Prism 9.

\section{MTS cell viability assay}

Cell viability was determined by the MTS cell viability assay as described previously [21, 23, 26-35]. In brief, 250-1000 cells were plated in the wells of a 96-well plate. Cells were then treated with DMSO, chemical inhibitors, or aCT1 peptides at the indicated doses for 6 days. For aCT1 treatment, we intended to plate cells at a low density to minimize the formation of gap junctions, and thus, more $\mathrm{Cx} 43$-hemichannels will be present. Because the half-life of aCT1 is about $48 \mathrm{~h}$, cells were replenished with fresh peptide every other day. MTS reagent was added and cell viability was determined by measuring the absorbance at $490 \mathrm{~nm}$ (MTS) using a FilterMax F3 microplate reader (Molecular Devices, LLC). Percent cell viability was obtained by dividing the absorbance of treatment groups to those of untreated and respective vehicle control groups.

\section{Caspase 3/7 activity assay}

Apoptosis was measured using the Caspase-Glo ${ }^{\circ} 3 / 7$ Assay (Promega) based on the manufacturer's instructions and our previous work [21, 27-29]. In brief, cells were plated at 250-1000 cells/well in 96-well plates and treated with drugs as described for 6 days. Caspase-Glo ${ }^{\circ}$ reagent was added and the luminescence was measured using a FilterMax F3 microplate reader.

\section{Immunoblotting}

Immunoblotting was performed as described previously [21, 23, $26-29,31,32,35-38]$. To prepare cell lysates from tumor tissues, lysis buffer (20 mM HEPES pH 6.8, $140 \mathrm{mM} \mathrm{NaCl}, 2.5 \mathrm{mM} \mathrm{MgCl} 2,2.5 \mathrm{mM} \mathrm{CaCl}_{2}$, $1 \%$ NP40, $0.5 \%$ sodium deoxycholate, protease inhibitor, and phosphatase inhibitors) was added to minced tissues followed by homogenization and protein quantification. $25-50 \mu \mathrm{g}$ total proteins from cell lysates were resolved on SDS-PAGE gels and transferred onto PVDF membranes. Antibodies were added and their dilution rates were summarized in Supplemental Table S1. Images of chemiluminescence from SuperSignal West Pico or Femto substrates (ThermoFisher) were taken using the ChemiDoc MP (BioRad). Whole images of immunoblotting were presented in the Supplemental Data-Whole blot images.

\section{Co-immunoprecipitation}

Co-immunoprecipitation was performed as previously described [37]. Cell pellets were lysed in lysis buffer described above. Total protein lysates were divided equally for each IP with input and IgG controls. Samples were incubated with primary antibodies overnight at $4{ }^{\circ} \mathrm{C}$ followed by Protein $\mathrm{G}$ Dynabeads. Antibodies and $\mathrm{lgG}$ dilutions were listed in Supplemental Table S1. Dilution rates vary due to recommendations from manufacturers and different concentrations of antibodies and IgG.

\section{Gene knockdown or expression}

Knockdown of CX43 or PI3K genes was described previously [21, 26, 27]. Information regarding short hairpin (sh) RNA of Cx43 or PI3K genes or constructs expressing gene mutants was included in the Supplemental Table S1. pcDNA3.2-GJA1 STOP was kindly provided by Dr. James Smyth at the Fralin Biomedical Research Institute (Roanoke, Virginia). In brief, lentiviral or retroviral vectors containing shRNAs or gene mutants were used to transfect HEK293T cells to generate viruses. Lentiviruses or retroviruses were subsequently used to transduce tumor cells. For transient transfection, 1-2 $\mathrm{gg}$ of plasmid DNA was transfected into cells using Effectene (QIAGEN). Transduced or transfected cells were subject to analyses of immunoblotting as described above.

\section{ATP/glutamate release}

ATP release was measured using the Kinase-Glo ${ }^{\oplus}$ Luminescent Kinase Assay (Promega). Glutamate release was measured using the Amplex ${ }^{T M}$ Red Glutamic Acid/Glutamate Oxidase Assay Kit (ThermoFisher). In brief, 1000 cells were plated in the wells of 96 -well plates followed by the indicated treatments. Media and cell lysates were then analyzed using the above kits according to the manufacturer's instructions.

\section{Bliss independence model}

Synergism was assessed by Bliss independence $[39,40]$, which is based on the null hypothesis that each drug acts independently without superadditive or antagonistic effects. The predicted effect was calculated using $\operatorname{Effect}(a+b)=E a+E b-E a E b$ for dual therapies and $\operatorname{Effect}(a+b+c)=$ $\mathrm{Ea}+\mathrm{Eb}+\mathrm{Ec}-\mathrm{EaEb}-\mathrm{EaEc}-\mathrm{EbEc}-\mathrm{EaEbEc}$ for triple therapies. The overall effect was presented as Excess Over Bliss (EOB) scores. EOB $>0 \%$ indicates a synergistic effect, whereas $\mathrm{EOB}=0 \%$ indicates an additive effect and $\mathrm{EOB}<0 \%$ indicates an antagonistic effect.

\section{Mouse experiments}

Mouse experiments were performed based on the methods described previously $[27,29,38]$, with modifications. All animal studies were approved by the Institutional Animal Care and Use Committee of Virginia Tech. In total, $2 \times 10^{6}$ SF-295 cells were mixed with Matrigel Matrix (Corning) and subcutaneously injected into the flanks of 8-week-old SCID/ beige mice (Taconic Biosciences). As we were measuring tumor sizes at different time points, 8 mice were assigned to each treatment group 8 days post-injection. The sample size was determined based on power analysis using $G^{*}$ Power. Mice were treated with drugs as indicated in the figure. No blinding was used. Drugs were administered every other day via intraperitoneal injection (TMZ and TGX-221) or through intratumoral injection (aCT1). Tumors were measured daily using a caliper. Sick mice or mice with $10 \%$ weight loss would be excluded. On day 18 , mice were 

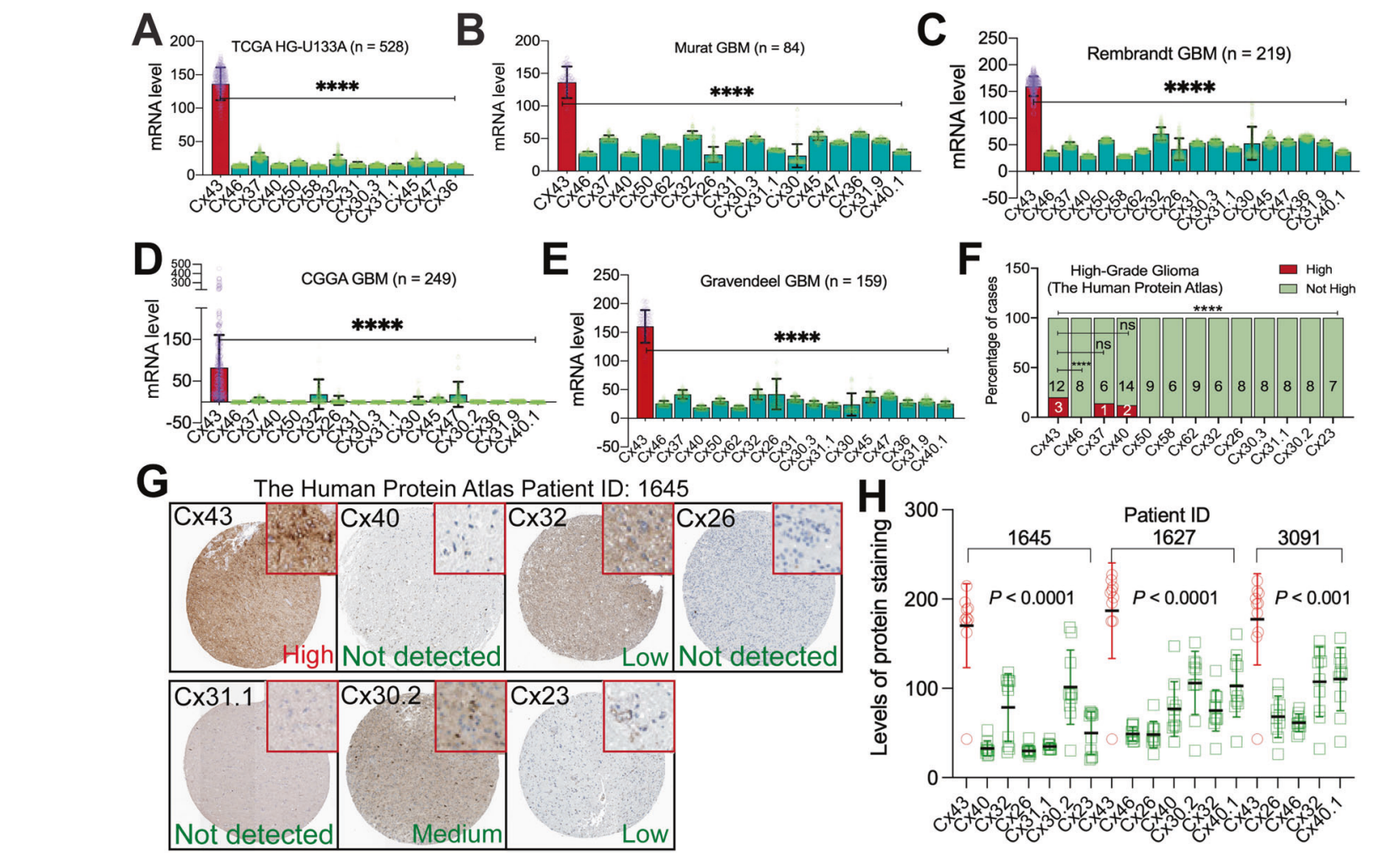

Fig. 1 Cx43 is expressed at the highest level among all connexins in a subpopulation of GBM. mRNA levels of connexins in GBMs from TCGA (A), Murat (B), Rembrandt (C), CGGA (D), and Gravendeel (E). Shown are average reads of microarray or RNAseq. Cx43 is presented as red bars with purple data points. Other connexins are labeled as green bars and yellow data points. F Staining scores of connexins in high-grade glioma. Case numbers with high (red) or not high (green) levels of connexins are shown. G Histological images of connexins in a high-grade glioma tumor. Inset images (highlighted in red) were cropped from original images in order to highlight immunostaining details. H Quantification of histological images from three GBM patients using Imaging J. Intensities of protein staining in 10 different cells were measured and quantified. GBM datasets were retrieved from cBioPortal, GlioVis, or CGGA data portal. Immunostaining results of high-grade glioma were obtained from the Human Protein Atlas. Statistical analyses: One-Way ANOVA with Dunnett test for correction of multiple comparisons and Fisher's exact test. ns not significant; ${ }^{* * * *} P<0.0001$. Error bars are standard deviations.

euthanized, and tumors were harvested. Tumor volumes $\left(\mathrm{mm}^{3}\right)$ were calculated using the formula: (length $\times$ width $\left.^{2}\right) / 2$.

\section{Statistical analyses}

Distribution and variation of data points in each experiment were analyzed and estimated. Three to four independent experiments were performed in cell-based assays, yielding standard deviations shown as error bars. Drug combinations were repeated in different cell lines. One-way ANOVA with Dunnett test for correction of multiple comparisons, Fisher's exact test, and Student's $t$ test were used to determine statistical significance and similarity of variance. All tests were two-sided. The center values shown in each figure were means.

\section{RESULTS}

Cx43, but not other connexins, is highly expressed in a subpopulation of GBM and mRNA levels of Cx43 correlate with poor prognosis and chemoresistance

Studies on the expression of $\mathrm{Cx} 43$ in normal brain and malignant glioma have shown discrepant results $[15,41-48]$. Despite that Cx43 mRNA levels are usually high in normal brain and low-grade glioma and vary in high-grade glioma, whether $\mathrm{Cx} 43$ and other connexins (Supplemental Table S2) are equally expressed in GBM and important for GBM chemoresistance has not yet been explored. To address this, we queried publicly available online GBM databases and analyzing programs, including TCGA (https:// www.cancer.gov/tcga), GlioVis [49], CGGA [50], and DepMap [51]. Cx43 mRNA was consistently expressed at the highest level among all connexins in primary GBM tumors from six different datasets (sample size from 84 to 526) and 54 GBM cell lines (Fig. 1A-E and Supplemental Fig. S1; $P<0.0001$ ). Based on immunostaining results from The Human Protein Atlas [52], levels of $\mathrm{C} \times 43$ protein in high-grade glioma were higher than other connexins, except Cx37 or Cx40 (Fig. 1F). In the same GBMs expressing high levels of $\mathrm{Cx} 43$ (Cx43-high), staining intensities of Cx43 were the highest compared to other connexins (Fig. 1G, H and Supplemental Fig. S2). This expression pattern was not found in low-grade glioma (Supplemental Fig. S3A). Intriguingly, Cx43 protein levels were low in glia cells in the cerebral cortex where GBMs are frequently found [53], despite that glia cells in basal ganglia and hippocampus expressed high levels of Cx43 (Supplemental Fig. S3B). Recent transcriptomic and proteomic analyses in the same high-grade glioma cell lines (DepMap) showed a strong correlation $(r=0.6 ; P=0.03)$ between mRNA levels and protein levels of Cx43 (Supplemental Fig. S3C) and that levels of Cx43 proteins were higher than those of other connexins (Supplemental Fig. S3D). Collectively, Cx43 mRNA and proteins are expressed at higher levels than other connexins in a subpopulation of GBMs.

Survival analyses (Fig. $2 A$ ) revealed that high levels of $\mathrm{C} \times 43$ mRNA were associated with poor prognosis of all GBM patients (All GBM), primary GBMs with promoter methylation of MGMT (MGMT-) [6], and recurrent GBMs (recurrent GBM). These results were consistent with our previous studies [21] and the finding that recurrent GBMs are often refractory to TMZ [5]. In contrast to these results, no correlation was found between Cx43 mRNA levels and the prognosis of primary or MGMT-expressing (MGMT+) GBMs (Supplemental Fig. S4A). Cox univariate analyses, which yield a hazard ratio $(H R)$ that determines chance of death $(H R>1$ 
A
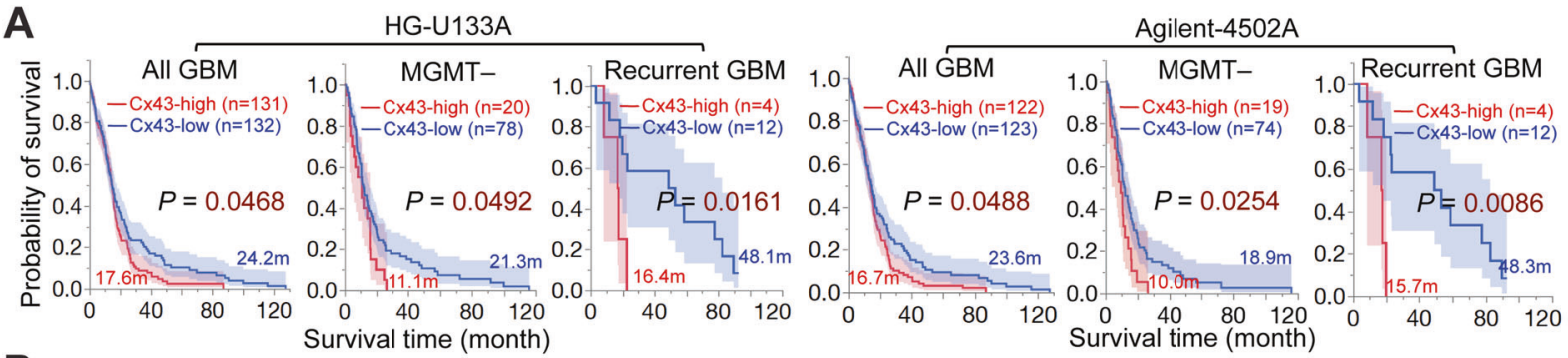

B HG-U133A

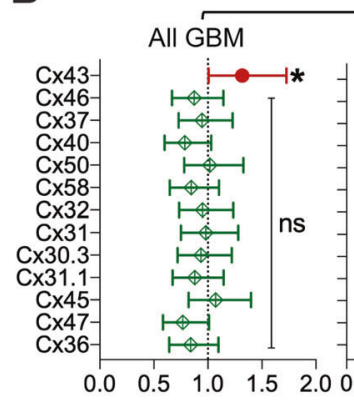

C

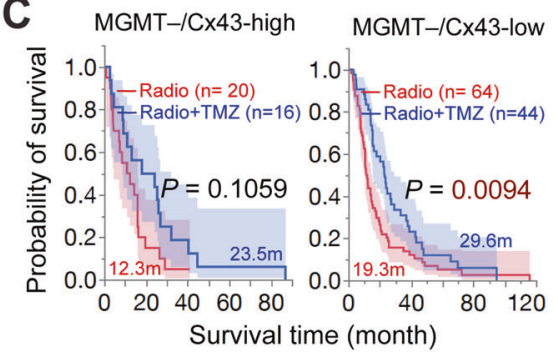

MGMT-

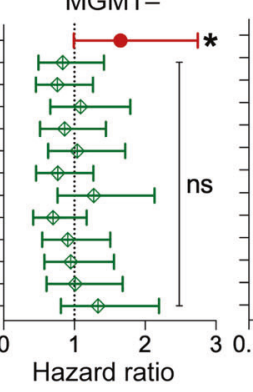

30.1
Recurrent GBM

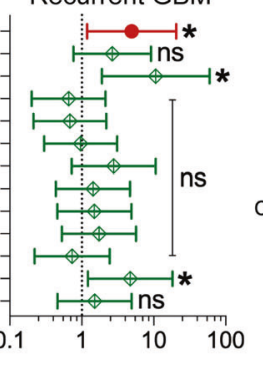

Agilent-4502A

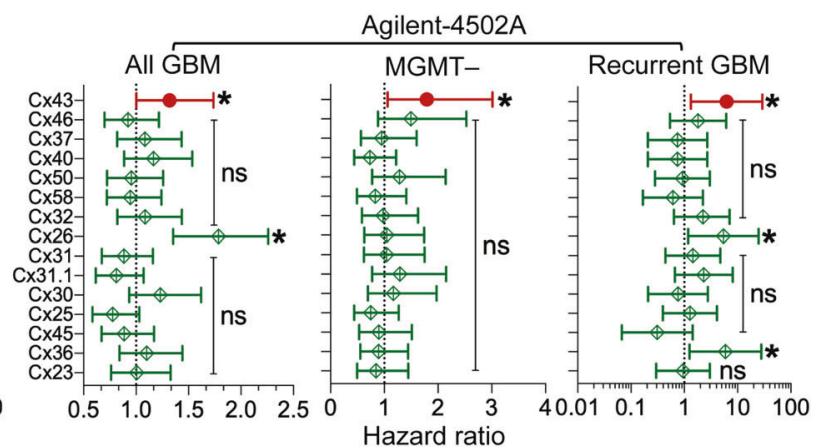

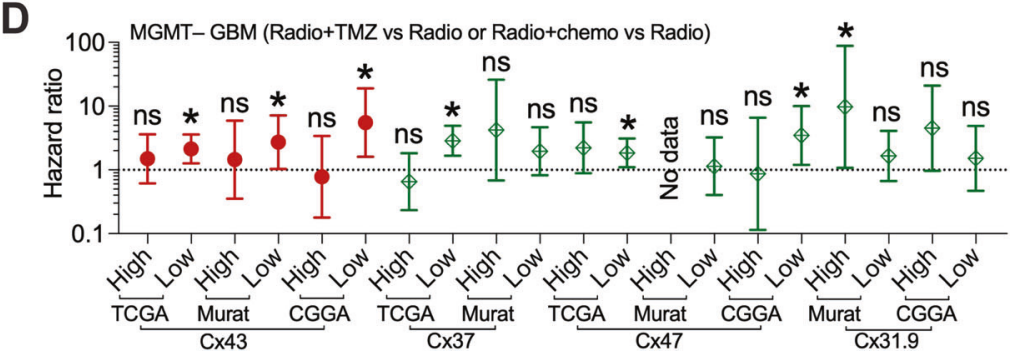

Fig. 2 mRNA levels of Cx43, but not other connexins, correlate with GBM poor prognosis and chemoresistance. GBM datasets were retrieved from cBioPortal, GlioVis, or CGGA data portal. Immunostaining results of high-grade glioma were obtained from the Human Protein Atlas. A Kaplan-Meier analysis in the TCGA HG-U133A and Agilent-4502A microarray datasets. Patients were divided into Cx43-high (red; top 25 percentile) or Cx43-low (blue; bottom 25 or 75 percentile) based upon Cx43 mRNA levels in all GBM (All GBM), MGMT promoter methylated primary GBM (MGMT-), or recurrent GBM only (Recurrent GBM). Case number ( $\mathrm{n})$, mean survival time in months ( $\mathrm{m})$, and log-rank $P$ values are shown. Red or blue shadows represent $95 \%$ confidence interval of Cx43-high or Cx43-low group, respectively. B Cox univariate analysis in the TCGA HG-U133A and Agilent-4502A microarray datasets. The Cox univariate analysis employs the Cox proportional hazards model to yield a hazard ratio that indicates risk levels of death in patients with high mRNA levels of connexins compared to those with low levels. The resulting $P$ value determines significance of hazard ratio. Cx43 is highlighted in red. C Kaplan-Meier analysis in TCGA HG-U133A. MGMT- GBMs were divided into Cx43-high or Cx43-low group as described above. Patients treated with radiation alone (Radio; red) were compared to patients treated with both radiation and TMZ (Radio+TMZ; blue). D Cox univariate analysis in TCGA HG-U133A, Murat GBM, and CGGA recurrent GBM. MGMT-deficient primary GBMs or recurrent GBMs were divided into Cx43-high or Cx43-low groups. One-Way ANOVA was used to determine statistical significance. ${ }^{*} P<0.05$. ${ }^{* * *} P<0.0001$. ns: not significant. Error bars are standard deviations.

indicates high risk of death), showed that Cx43-high patients displayed high risk of death in the group of All GBM, MGMT-, and Recurrent GBM (Fig. 2B), but not in the group of primary or MGMT + GBM (Supplemental Fig. S4B). Other connexins, however, had variable HRs exhibiting either no statistical significance or not consistently significant in all three groups. Similar results were found in Murat GBM (Supplemental Fig. S5) and recurrent GBMs from CGGA (Supplemental Fig. S6). Our results demonstrate that mRNA levels of Cx43, but not other connexins, correlate with the prognosis of MGMT- or recurrent GBMs.

TMZ improves GBM prognosis when used in combination with radiation [6]. However, the combination of radiation and TMZ (Radio+TMZ) or chemo (Radio+chemo) showed no difference in the survival of Cx43-high/MGMT- GBMs (Fig. 2C, D and Supplemental Fig. S7), whereas the addition of TMZ or chemo increased the survival of MGMT- GBMs expressing low levels of Cx43 mRNA (Cx43-low). Of note, high levels of Cx37, Cx47, or Cx31.9 did not show similar results (Fig. 2D). In line with our prior studies [21, 41], it is suggested that GBM patients with high levels of Cx43 mRNA are resistant to chemotherapies.

\section{Cx43 confers resistance to TMZ by activating PI3K}

Next, we explored how $\mathrm{Cx} 43$ confers TMZ resistance. We have previously shown that the $\mathrm{Cx} 43$ peptide inhibitor aCT1 inactivates $\mathrm{PI3K}$ [21], leading us to hypothesize that CX43 activates PI3K to induce TMZ resistance. To test this hypothesis, we treated Cx43-high/ TMZ-resistant U87MG cells with TMZ or aCT1. aCT1 blocked phosphorylation of Cx43 at serine 368 (Fig. 3A, pCx43-S368), an indicator of Cx43 activity [54]. As expected, aCT1 significantly decreased the phosphorylated form of AKT serine/threonine kinase (pAKT-S473; Fig. 3A and Supplemental Fig. S8A). Previous research $[55,56]$ has suggested that $C \times 43$ regulates the activity of the mitogen-activated protein kinase (MAPK) pathway, including the RAF proto-oncogene serine/threonine-protein kinase (RAF)/extracellularsignal-regulated kinase (ERK) cascade and the SRC proto-oncogene non-receptor tyrosine kinase (SRC) pathway. aCT1 modestly reduced levels of pcRAF-S338, pERK-T202/T204, or pSRC-Y416 (Fig. 3A). To corroborate results from aCT1, we knocked down Cx43 using an shRNA. Cx43 shRNA decreased levels of Cx43 and pCx43-S368 and inactivated PI3K/AKT in U87MG cells, but not in Cx43-low A172 cells having low levels of pAKTs (Fig. 3B and Supplemental Fig. S8B). 
A

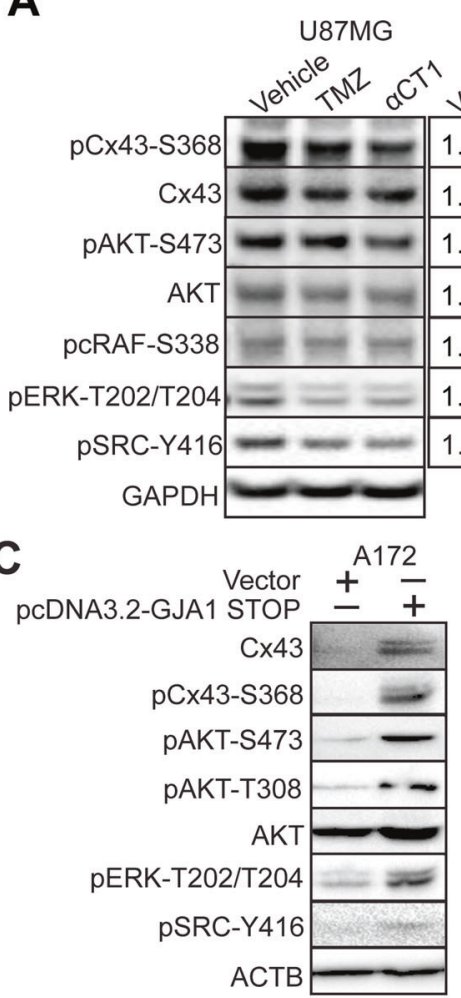

$\mathbf{F}$

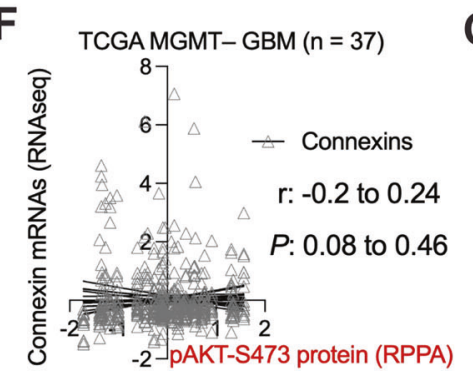

$\mathrm{I} \square$ pBABE $\square$ рBABE-PIK3CA-E545K $\mathrm{J}$

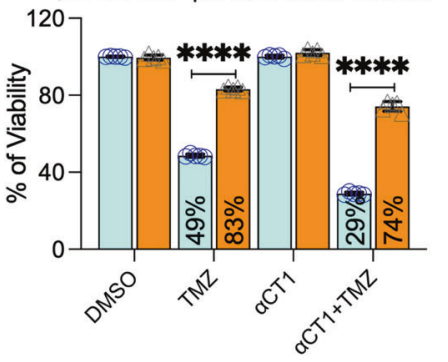

B

\begin{tabular}{|c|c|c|c|c|c|c|c|c|}
\hline 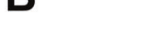 & U8 & $7 M G$ & & 72 & & $7 \mathrm{MG}$ & & 172 \\
\hline shRNA: & $n^{5}$ & $\mathrm{ct}^{\mathrm{A}^{2}}$ & S & $\mathrm{cot}^{+\mathrm{A}^{\prime}}$ & NS & $\mathrm{c}^{+x^{3}}$ & NS & $c^{+4}$ \\
\hline Cx43 & 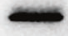 & $\longrightarrow$ & -2. & & 1.0 & 0.1 & 0.1 & 0.0 \\
\hline pCx43-S368 & 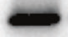 & - & - & & 1.0 & 0.2 & 0.1 & 0.0 \\
\hline$p 110 \alpha$ & & & Anstos & & 1.0 & 1.0 & 1.1 & 1.0 \\
\hline $\mathrm{p} 110 \beta$ & & & - & - & 1.0 & 1.0 & 0.4 & 0.3 \\
\hline p110ס & - & - & 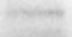 & & 1.0 & 0.5 & 0.2 & 0.1 \\
\hline pAKT-S473 & $=$ & - & & & 1.0 & 0.3 & 0.0 & 0.0 \\
\hline pAKT-T308 & & & & & 1.0 & 0.4 & 0.0 & 0.0 \\
\hline AKT & & 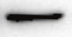 & - & - & 1.0 & 0.6 & 0.2 & 0.2 \\
\hline
\end{tabular}

D

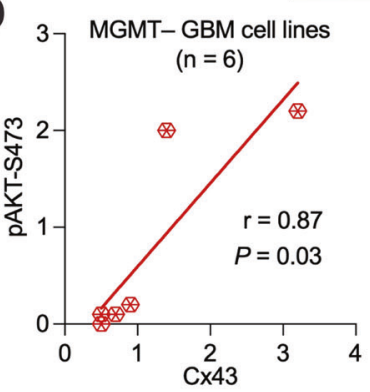

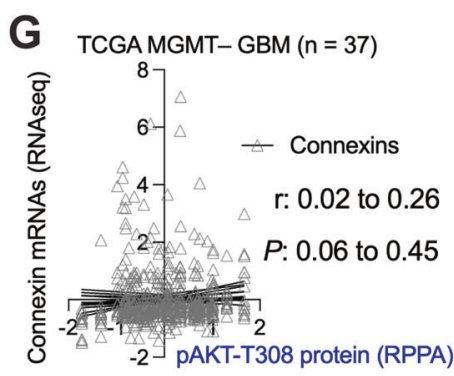
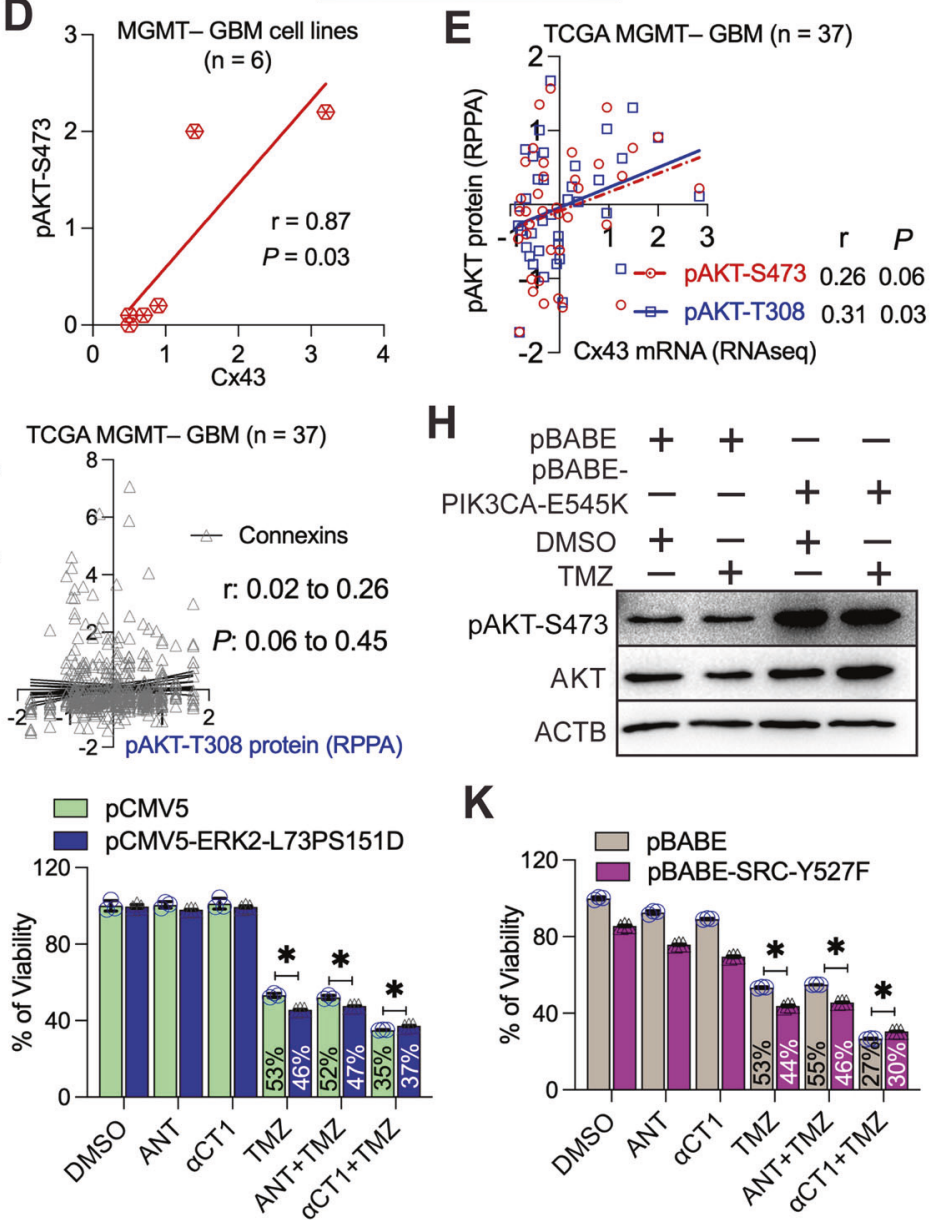

K

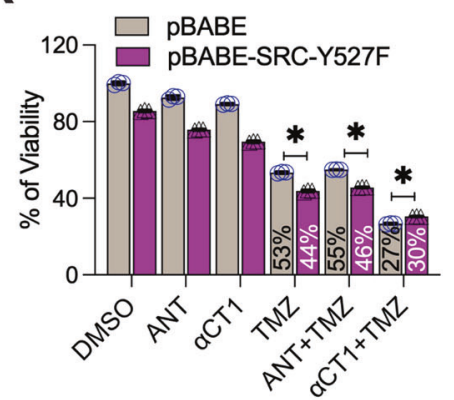

Moreover, ectopically expressing Cx43 in A172 cells activated PI3K/ AKT and ERK (Fig. 3C). These results have demonstrated that Cx43 regulates PI3K/AKT activity in GBM. Through reanalyzing data from our previous work [21, 27], we detected a strong correlation between Cx43 and pAKT-S473 in six MGMT- GBM cell lines (Fig. 3D and Supplemental Table S2). A positive trend was also found between levels of Cx43 mRNA and pAKT-S473 or PAKT-T308 in 37 MGMTGBM patients in the TCGA dataset (Fig. 3E). Other connexins, however, failed to show statistically significant correlations with either pAKT-S473 (Fig. 3F) or pAKT-T308 (Fig. 3G).

To determine whether $\mathrm{PI} 3 \mathrm{~K}$ is required for aCT1-medicated chemo-sensitization, we overexpressed PIK3CA-E545K, a PI3K mutant that constitutively activates PI3K, in U87MG cells (Fig. $3 \mathrm{H}$ ). PIK3CA-E545K counterbalanced the growth inhibition induced by $\mathrm{TMZ}$ or by a combination of $\mathrm{TMZ}$ and $\mathrm{aCT} 1$ (Fig. 3l). This counteraction was not seen in U87MG cells expressing an active mutant of ERK (ERK2-L73PS151D; Fig. 3J) or SRC (SRC-Y527F; Fig. 3K). These results suggest that the inhibition of PI3K is important for aCT1 to restore $T M Z$ sensitivity.

\section{aCT1 inactivates PI3K independent of Cx43-channels and} Cx43 selectively binds to a PI3K catalytic subunit

Because the Cx43-CT regulates the activity of Cx43-channels [57], it is possible that small molecules such as ATP or glutamate 
Fig. 3 Cx43 blockade inactivates PI3K. A Signaling pathways affected by $\alpha C T 1$. Cx43-high U87MG cells were treated with $100 \mu \mathrm{M} \alpha \mathrm{CT} 1$ or $50 \mu \mathrm{M}$ TMZ for 4 days. pAKT-S473, pcRAF-S338, pERK-T202/T204, and pSRC-Y416 were analyzed using immunoblotting. Glyceraldehyde 3-phosphate dehydrogenase (GAPDH) was the loading control. Band intensities (mean gray value $\times$ area) were quantified using Image J. The vehicle DMSO or water was set as 1.0 and each treatment was normalized to the vehicle. B PI3K signaling upon depletion of CX43. U87MG and Cx43-low A172 cells were treated with a non-silencing short hairpin RNA (NS shRNA) or a Cx43 shRNA. Band intensities were quantified using Image J. U87MG cells treated with NS shRNA were set as 1.0. $\beta$-actin (ACTB) was the loading control. C PI3K signaling in Cx43-overexpressing cells. U87MG cells were transfected with either vector or pcDNA3.2-GJA1 STOP. PI3K, ERK, and SRC signaling was analyzed using immunoblotting. Pearson coefficient correlation analysis between protein levels of Cx43 and pAKT-S473 was performed using Prism 9 in 6 MGMT- GBM cell lines (D), mRNA levels of Cx43 and protein levels of pAKT-S473 or pAKT-T308 in MGMT- patients (E), or mRNA levels of connexins and protein levels of pAKT-S473 (F) and pAKT-T308 (G) in MGMT- GBMs. The Pearson correlation coefficient $(r)$ and $P$ value that determines statistical significance of the coefficient are shown. Cell line data were retrieved from our previous studies [21, 27]. H Expression of PIK3CA-E545K (an active PI3K mutant). U87MG cells were transfected with pBABE or pBABE-PIK3CA-E545K encoding PIK3CA-E545K followed by the treatment of $100 \mu \mathrm{M}$ TMZ. DMSO is the vehicle control. I The effect of PIK3CA-E545K on the $\alpha C T 1 / T M Z$-induced growth inhibition. The above-transfected cells were treated with a combination of $100 \mu \mathrm{M} \alpha \mathrm{CT} 1$ and/or $100 \mu \mathrm{M} \mathrm{TMZ}$ for 6 days. Cell viability was measured using the MTS viability assay. Percentages of viability were obtained by normalizing the MTS readings of treatment groups to that of DMSO. J The effect of ERK2-L73PS151D on the $\alpha$ CT1/TMZ-induced growth inhibition. U87MG cells were transfected with pCMV5 or pCMV5-ERK2-L73PS151D (encoding an active ERK2 mutant) followed by the treatment of $\alpha C T 1$ or antennapedia peptide (ANT; the control peptide for $\alpha C T 1$ ) and/or TMZ. K The effect of SRC-Y527F on the $\alpha C T 1 / T M Z$-induced growth inhibition. U87MG cells were transfected with pBABE or pBABE-SRC-Y527F (encoding an active SRC mutant) followed by the treatment of $\alpha C T 1$ or ANT and/or TMZ. RNA sequencing (RNAseq) data and results of reverse phase protein array (RPPA) were retrieved from the TCGA database. Student's $t$ test was used to determine statistical significance. ${ }^{*} P<0.05$; $* * * * P<0.0001$. Three independent experiments were performed to yield standard deviations (error bars).

released from Cx43-channels activate PI3K in GBM cells as they do in astrocytes [58]. To test this possibility, we treated U87MG cells with Gap27, a Cx43 peptide inhibitor that targets the second extracellular loop of Cx43 and blocks Cx43-channels [59]. Gap27, however, slightly increased AKT activity (Fig. 4A). Next, we examined the levels of ATP or glutamate in Cx43-high SF295 and LN229/GSC as well as Cx43-low LN229 cells. Regardless of levels of Cx43, levels of ATP or glutamate in culture media either elevated or remained unchanged in aCT1-treated cells (Fig. 4B-D). This could be explained by the enhanced permeability of $\mathrm{Cx} 43$ hemichannels upon dephosphorylation of $\mathrm{Cx} 43$ by aCT1 (Fig. 3A) [60]. ATP or glutamate levels remained unchanged in cells (Fig. 4E, F). Our results suggest that $\mathrm{Cx} 43$-channels are dispensable for PI3K activation in GBM cells.

Cx43-CT interacts with certain signaling molecules [55]. It is likely that $\mathrm{C} \times 43$ binds to PI3K catalytic subunits to activate PI3K. The Class I PI3K family consists of four highly homologous catalytic subunits: PI3K catalytic subunits $a, \beta, \delta$, and $\gamma$ (PIK3CA, PIK3CB, PIK3CD, and PIK3CG) encoding p110a, p110 $\beta, p 110 \delta$, and p110 $\gamma$, respectively [61]. Our previous work has demonstrated that PI3K catalytic subunits play divergent roles in GBM cell survival, with p110 $\beta$ being the most dominant isoform in GBM [27]. To determine whether PI3K catalytic subunits also function divergently in Cx43-induced PI3K activation, we reanalyzed protein expression data in six MGMT- GBM cell lines (Supplemental Table S3). Levels of $\mathrm{Cx} 43$ protein showed a positive and statistically significant correlation with those of $p 110 \beta$, but not other p110s or the regulatory subunit p85 (Fig. 4G). mRNA levels of $\mathrm{Cx} 43$ also positively corresponded with those of $\mathrm{PIK} 3 \mathrm{CB}$, but not other PI3K subunits, in 89 MGMT- GBM patients (Fig. 4H). In the same GBM patients, PIK3CB displayed no or negative correlation with other connexins, except $\mathrm{C} \times 31$ (Fig. 4I). Such a positive correlation between Cx43 mRNA and PIK3CB mRNA was recapitulated in multiple GBM datasets (Supplement Fig. S9) and further verified by the finding that GBMs with high levels of pAKT-S473 or $p 110 \beta$, but not other $\mathrm{p} 110 \mathrm{~s}$, were resistant to TMZ indicated by increased TMZ IC50s (Fig. 4J, K). To further probe the molecular details of Cx43-induced $\mathrm{PI} 3 \mathrm{~K}$ activation, we monitored protein-protein interactions between $\mathrm{Cx} 43$ and p110 proteins. $\mathrm{Cx} 43$ was coprecipitated with $\mathrm{p} 110 \beta$ (Fig. $4 \mathrm{~L}$ ) but not with $\mathrm{p} 110 \mathrm{a}$ or $\mathrm{p} 110 \delta$ (Fig. 4M, N), demonstrating a selective binding between $\mathrm{C} \times 43$ and $\mathrm{p} 110 \beta$. We did not examine $\mathrm{p} 110 \mathrm{\gamma}$ because this subunit is not detectable in GBM [27]. To determine whether aCT1 binds to Cx43 and/or $\mathrm{p} 110 \beta$, we treated U87MG cell lysates with aCT1 and found that aCT1 was pulled down together with $\mathrm{p} 110 \beta$ and $\mathrm{C} \times 43$ (Fig. 4O). In the presence of $a C T 1$, more $\mathrm{p} 110 \beta$ was found in the
Cx43 precipitate. This might be because the $\mathrm{Cx} 43$ antibody is able to precipitate aCT1- and Cx43-bound protein complexes. Taken together, aCT1 inactivates PI3K independent of Cx43-channels and Cx43 selectively binds to $\mathrm{p} 110 \beta$.

\section{A combination of $\alpha C T 1$ and p110 $\beta$-selective inhibitors overcomes $\mathrm{TMZ}$ resistance}

aCT1 alone increases the sensitivity of LN229/GSC xenograft tumors to TMZ [21]; however, the short half-life of aCT1 demands high concentrations and repeated drug delivery, which may limit its therapeutic potential. Prompted by the above results, we tested the combination of $\mathrm{aCT} 1$ and $\mathrm{p} 110 \beta$-selective inhibitors in cultured cells and in mice. To achieve a synergistic therapeutic effect of multiple drugs, we optimized the dose of each individual drug in U87MG cells. By varying doses of TMZ or a p110 -selective inhibitor TGX-221 (TGX), we found that the double combination of $50 \mu \mathrm{M}$ TMZ and $20 \mu \mathrm{M}$ TGX-221 only reduced the viability of U87MG cells by $\sim 50 \%$ (Supplemental Fig. S10A, B). However, the addition of aCT1 as low as $10 \mu \mathrm{M}$ greatly increased the cytotoxic effect of the TMZ/TGX-221 double combination (Supplemental Fig. S10C). Next, we assessed synergistic drug effects using the Bliss independent model, a method commonly used to measure drug synergy $[39,40]$. This model yields Excess Over Bliss (EOB) scores. $E O B>0 \%$ indicates a synergistic effect; $E O B=0 \%$ means an additive drug effect; $E O B<0 \%$ refers to an antagonistic effect. 2.5-10 $\mu \mathrm{M}$ aCT1 together with TMZ/TGX-221 only yielded a weak synergistic effect on cell viability, whereas 12.5 to $50 \mu \mathrm{M}$ aCT1 exhibited a much stronger synergistic inhibition on cell viability (Supplemental Fig. S10D).

Based on these results, $30 \mu \mathrm{M}$ aCT1, $20 \mu \mathrm{M}$ TGX-221, and $50 \mu \mathrm{M}$ TMZ were used in a triple combination named aCT1/TGX/TMZ combo. The aCT1/TGX/TMZ combo synergistically reduced the viability of MGMT- /TMZ-resistant SF295, VTC-103, and VTC-003 cells (Fig. 5A and Supplemental Fig. S11A) that express high levels of Cx43 and p110 $[21,27]$. Notably, VTC-103, VTC-003, and other VTC lines described hereafter were derived from freshly dissected GBM tumors [21, 27]. EOB scores of the aCT1/TGX/TMZ combo were significantly higher than those of double combinations (Fig. 5B and Supplemental Fig. S11B). This synergistic effect was, however, not found in MGMT-/TMZ-sensitive LN229 and A172 or MGMT-/TMZ-resistant VTC-001 and VTC-005 (Fig. 5C, D and Supplemental Figs. S11C, D and S12) whose levels of Cx43 and p110 $\beta$ are low $[21,27]$. The aCT1/TGX/TMZ combo synergistically activated apoptosis in VTC-103 cells (Fig. 5E, F), whereas apoptosis was not synergistically induced in VTC-001 cells. To verify our in vitro studies in vivo, we treated mice bearing SF295 xenograft 
A

B

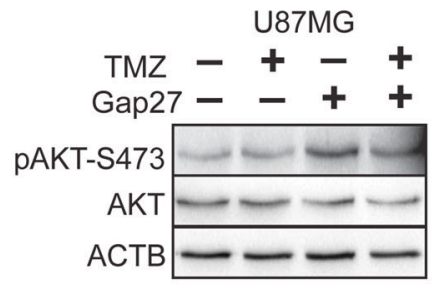

D

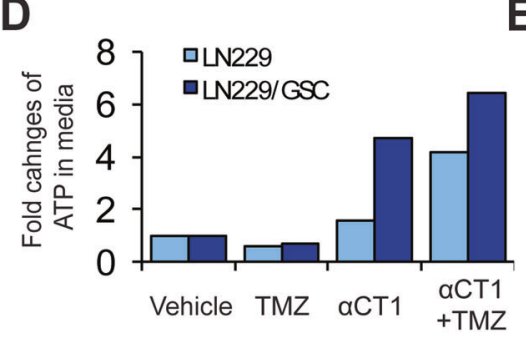

G

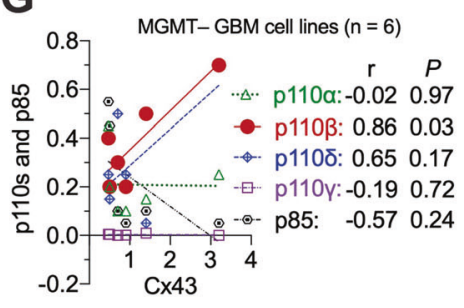

J

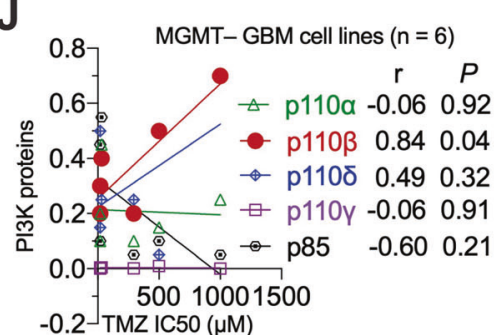

M

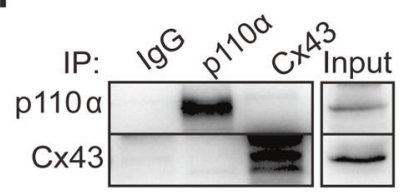

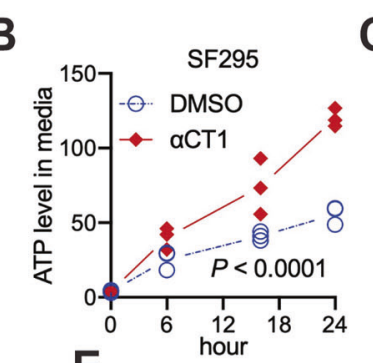

E

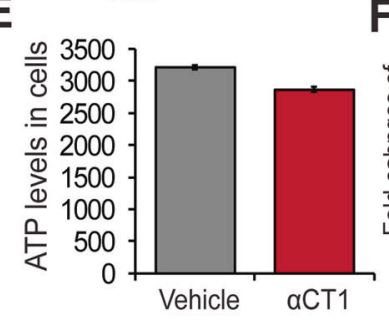

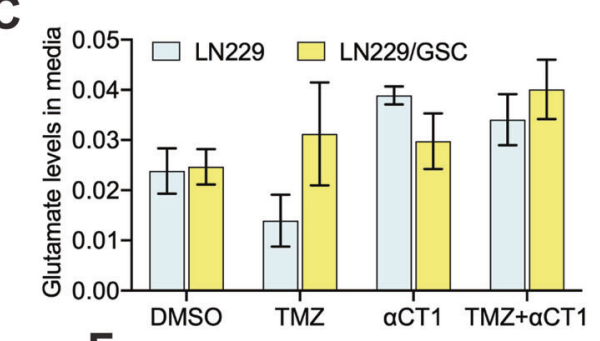

F

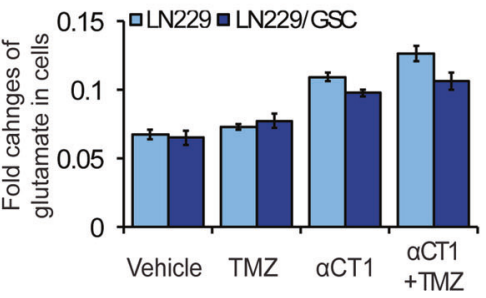

H

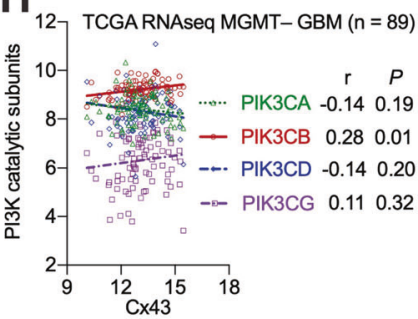

K $\quad{ }_{1000}$ MGMT-GBM $\square$

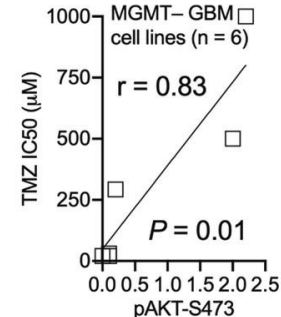

$\mathbf{N}$

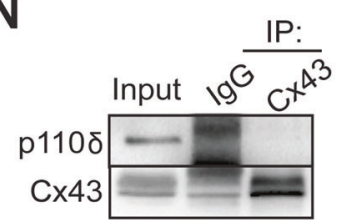

I

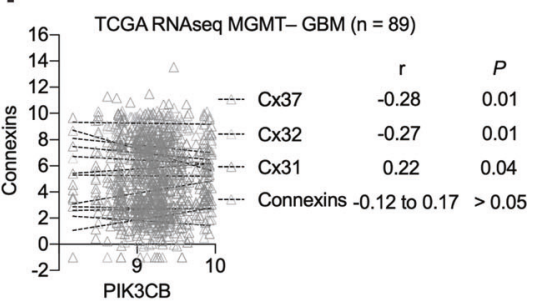

$\mathbf{L}$
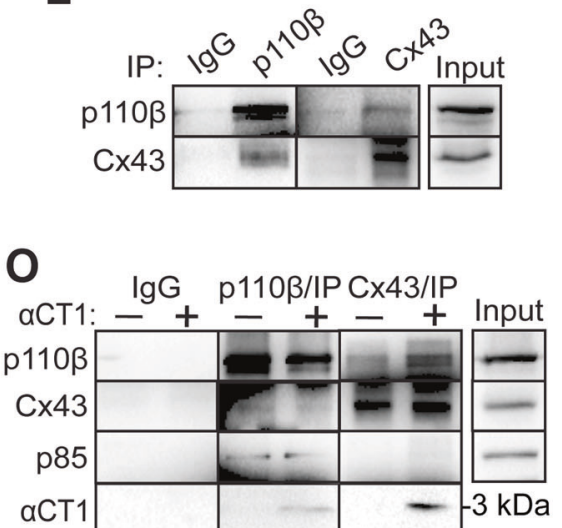

Fig. 4 aCT1 inactivates PI3K independent of Cx43-channels and Cx43 selectively binds to p110 U87MG cells were treated with $100 \mu \mathrm{M}$ TMZ or $100 \mu \mathrm{M}$ Gap27. PI3K signaling was assessed by immunoblotting. B ATP release from Cx43-high/ MGMT-/TMZ-resistant SF295 cells. Cells were treated with $100 \mu \mathrm{M} \alpha \mathrm{CT} 1$. Culture media were collected at different time points. ATP was measured using a colorimetric assay as described in Methods. One-way ANOVA was used to determine statistical significance. C Glutamate release in Cx43-low/MGMT-/TMZ-sensitive LN229 or Cx43-high/MGMT-/TMZ-resistant LN229/GSC cells. Cells were treated with $100 \mu$ M TMZ and/or $100 \mu \mathrm{M} \alpha \mathrm{CT} 1$. Glutamate in culture media was determined using a colorimetric assay. D ATP release in LN229 and LN229/GSC cells. E ATP levels inside of SF295 cells. F Glutamate levels inside of LN229 and LN229/GSC cells. GSC glioblastoma stem cells. Pearson coefficient Correlation between protein levels of Cx43 and PI3K catalytic subunits in 6 MGMT- GBM cell lines (G), mRNA levels of Cx43 and PI3K catalytic subunits in MGMT- GBM patients (H), mRNA levels of PIK3CB and connexins in MGMT- GBM patients (I), protein levels of p110 proteins and IC50s of TMZ in 6 MGMT- GBM cell lines (J), or protein levels of pAKT-S473 and IC50s of TMZ in 6 MGMT- GBM cell lines (K). Cell line data were retrieved from our previous studies $[21,27]$. RNAseq data were retrieved from the TCGA database. The Pearson correlation coefficient $r$ and

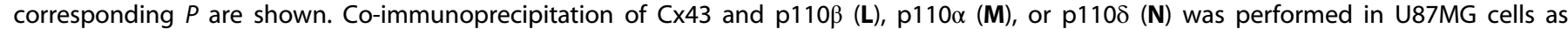
described in Methods. $\mathbf{O}$ Co-immunoprecipitation of Cx43 and p110 $\beta$ in U87MG cell lysates treated with $100 \mu \mathrm{M} \alpha \mathrm{CT} 1 . \alpha C T 1$ is about $3 \mathrm{kDa}$ and recognized by the Cx43 antibody. IP immunoprecipitation. Rabbit IgG was used as the control. Three independent experiments were performed to yield standard deviations (error bars).

tumors with $7.5 \mathrm{mg} / \mathrm{kg} \mathrm{TMZ} \mathrm{[21]} \mathrm{and} 20 \mathrm{mg} / \mathrm{kg}$ TGX-221 [27] through intraperitoneal injection in conjunction with $0.6 \mathrm{mg} / \mathrm{kg}$ aCT1 (equivalent to $30 \mu \mathrm{M}$ ) through intratumoral injection. The aCT1/TGX/TMZ combo (red line) stopped tumor growth (Fig. 5G, $P<0.05$ ), whereas double combinations (green, yellow, purple, or blue line) exhibited limited to no inhibition. While EOB scores of the triple combo increased over time, culminating on day 18 (Fig. 5H), Immunoblotting verified that aCT1 and TGX-221 together significantly decreased AKT phosphorylation (Fig. 5I). This confirmed a strong synergy amongst aCT1, TGX-221, and TMZ 
A

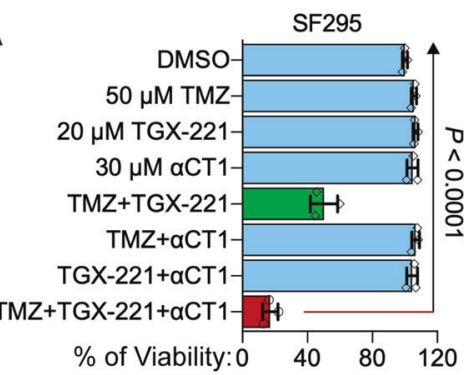

C

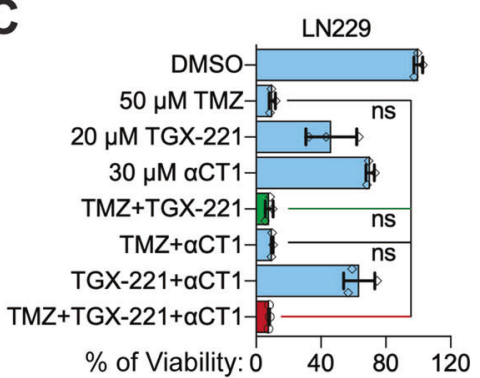

E
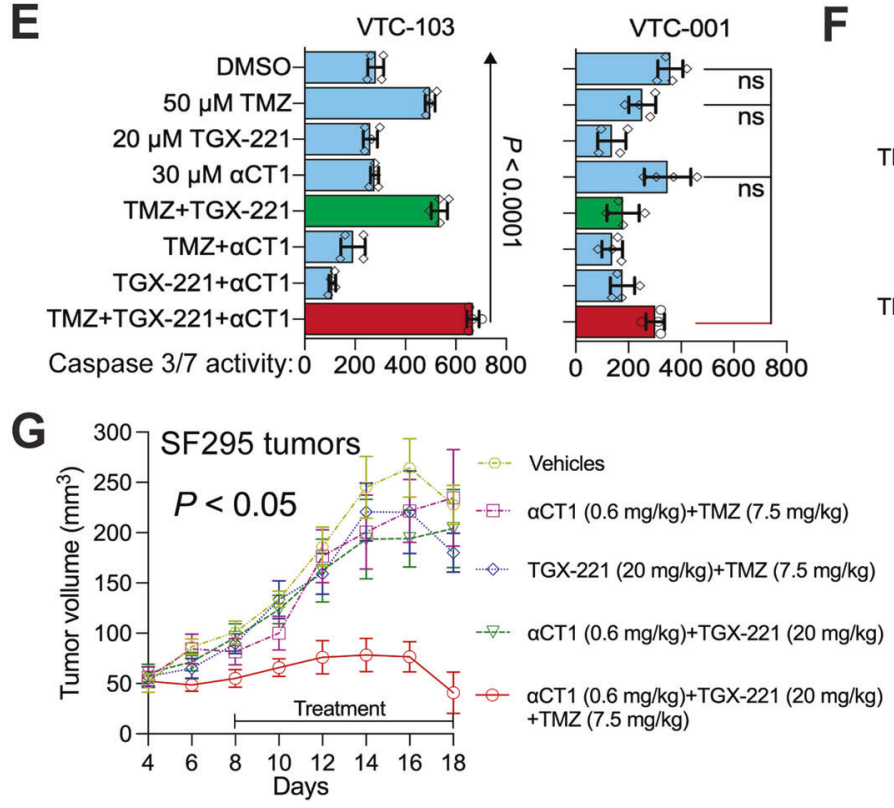

B

D

$\mathbf{F}$
Caspase 3/7 activity: 0200400600800
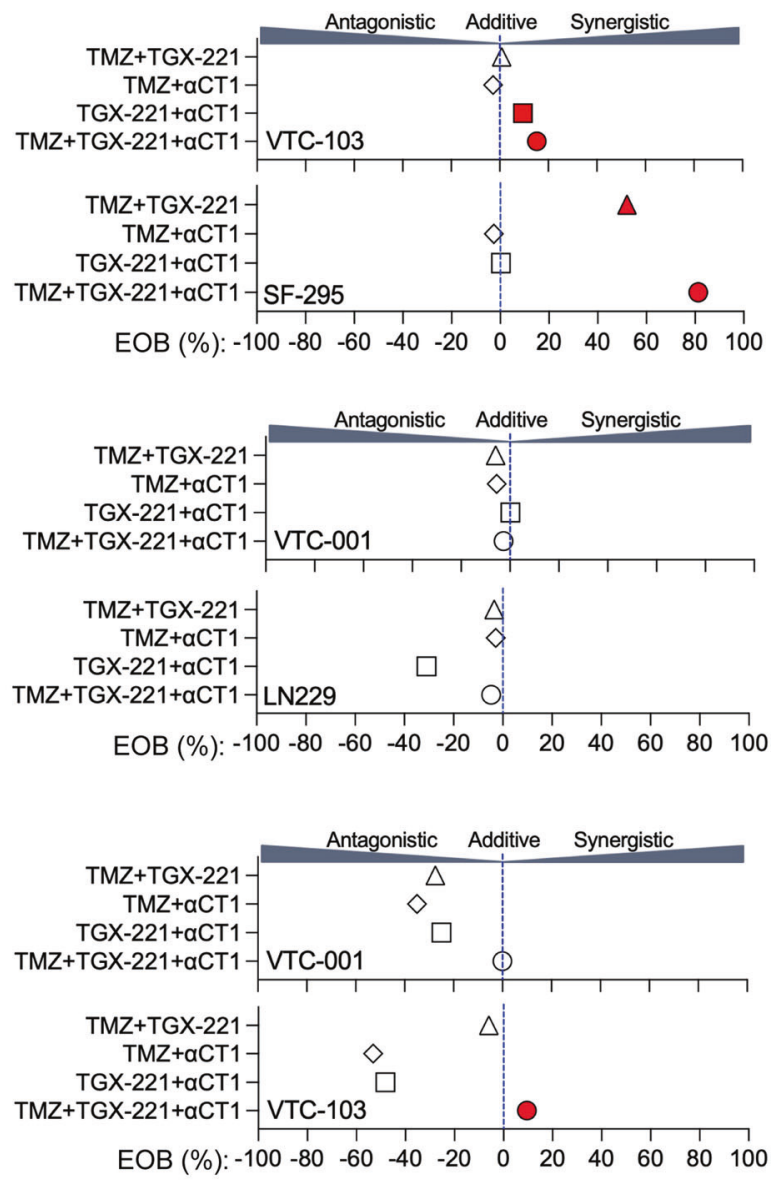

H
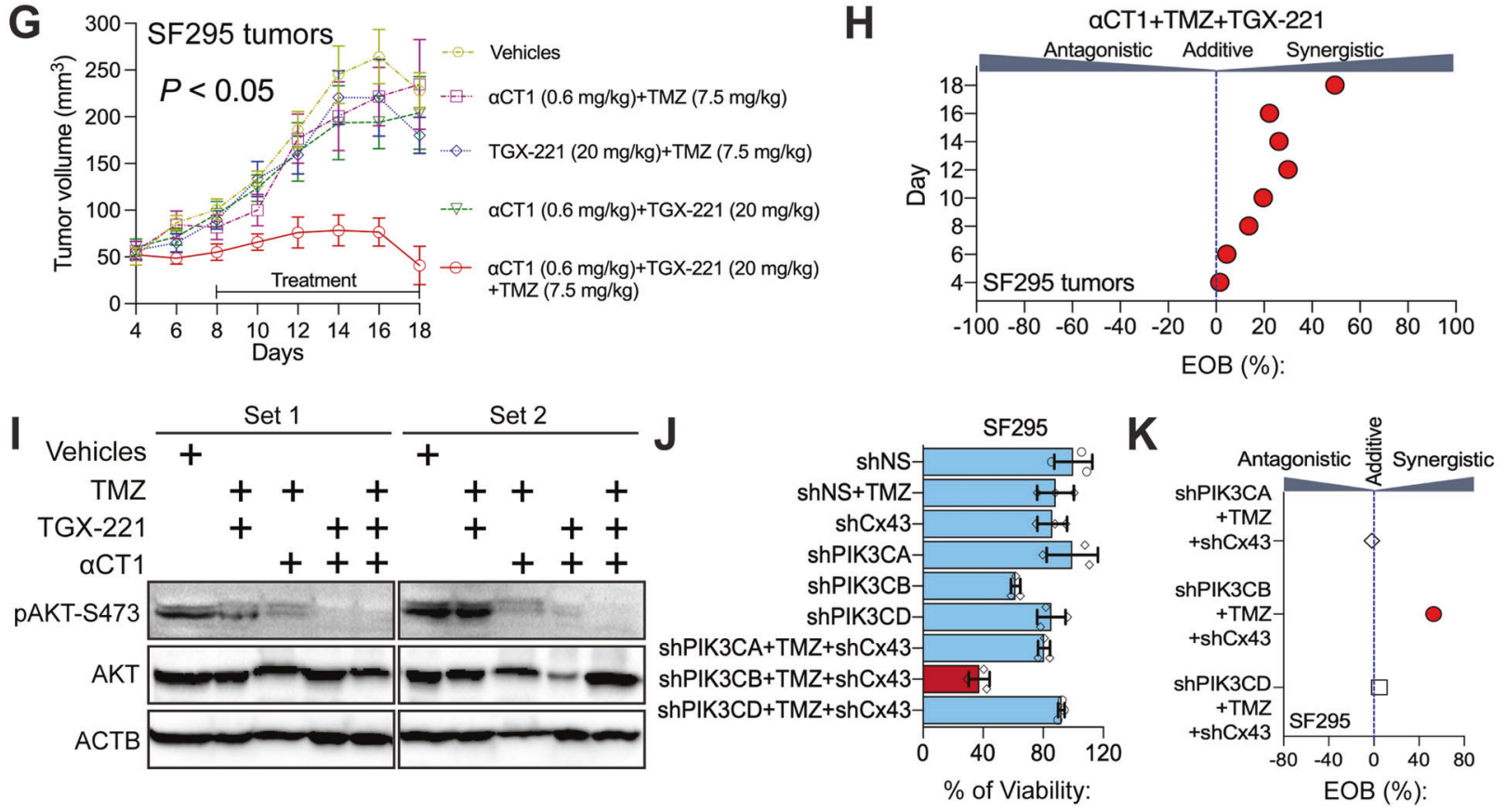

in vivo. To verify that the synergistic cytotoxicity is due to the blockade of $\mathrm{Cx} 43 / \mathrm{p} 110 \beta$, we knocked down $\mathrm{C} \times 43$ and individual PI3K catalytic subunits using shRNAs. Depletion of $\mathrm{p} 110 \beta$, but not p110a or p1108, blocked the growth of SF295 cells (Fig. 5J) and only the combination of PIK3CB shRNA, Cx43 shRNA, and TMZ yielded synergistic inhibition of cell viability (Fig. $5 \mathrm{~K}$, red circle).
To corroborate results from TGX-221, we tested another p110ß-selective inhibitor GSK2636771 (GSK), which has been used in a clinical study [62]. aCT1/GSK/TMZ combo entailing $25 \mu \mathrm{M}$ GSK2636771, $30 \mu \mathrm{M}$ aCT1, and $50 \mu \mathrm{M}$ TMZ synergistically blocked the viability of VTC-103 cells and U87MG cells, but not the viability of LN229 cells (Supplemental Fig. S13). aCT1/GSK/TMZ has 
Fig. 5 A combination of aCT1 and TGX-221 overcomes TMZ resistance in vitro and in vivo. A The effect of the $\alpha C T 1 / T G X / T M Z$ combo in

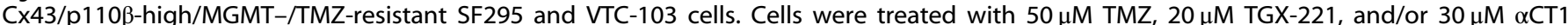
including single agents, double combinations, and the $\alpha \mathrm{CT} 1 / \mathrm{TGX} / \mathrm{TMZ}$ combo. This scheme has been repeated in experiments presented hereafter. Cell viability was determined using the MTS viability assay. Percentage of cell viability was obtained by normalizing the MTS reading of treatment groups to that of the DMSO group. B EOB scores were calculated using the Bliss Independence model. The drug combination is synergistic if EOB is more than $0 \%$, additive if EOB equals to $0 \%$, or antagonistic if EOB is less than $0 \%$. C The effect of the $\alpha C T 1 / T G X / T M Z$ in Cx43/p110ß-low/MGMT-/TMZ-sensitive LN229 and TMZ-resistant VTC-001 cells. D EOB scores of drug combinations in LN229 and VTC-001 cells. E Caspase 3/7 activity in VTC-103 and VTC-001 cells. The activity of cleaved caspase 3/7 (active) was determined using a luminescence assay as described in Methods. Shown are luminescence readings. F EOB scores of drug combinations in VTC-103 and VTC-001 cells. G The effect of $\alpha$ CT1/TGX/TMZ combo on SF295 xenograft tumors. SF295 cells were subcutaneously injected into immuno-deficient mice. 8 days later, mice were treated with TMZ, TGX-221, or $\alpha \mathrm{CT} 1$ through intraperitoneal or intratumoral injection every other day until day 18 . Tumor volumes are shown. H EOB scores of drug combinations in SF295 tumors at different days. I Immunoblotting of PI3K signaling in SF-295 tumors. Proteins were directly extracted from homogenized tumor tissues and analyzed using western-blotting as described in Methods. ACTB is the loading control. Two repeated experiments are shown. J The effect of shRNA of Cx43 or PI3K catalytic subunits on the TMZ sensitivity of SF295 cells. Cells were transfected with NS shRNA or shRNA of Cx43, PIK3CA, PIK3CB, or PIK3CD followed by the treatment of $50 \mu \mathrm{M}$ TMZ. Cell viability was determined using the MTS viability assay. Percentages of cell viability were obtained by normalizing the MTS readings of treatment groups to that of shNS group. K EOB scores of drug combinations in SF295 cells. One-way ANOVA with Dunnett test for correcting multiple comparisons or Student's $t$ test was used to determine statistical significance. ${ }^{*} P<0.05$; ns not significant. Drug combinations with strong synergistic effect were marked in red. Three independent experiments were performed to yield standard deviations (error bars).

achieved the same synergistic inhibition of GBM cell viability as the aCT1/TGX/TMZ combo. To determine the toxicity of these combinations on normal cells, we treated astrocytes with aCT1/ TGX/TMZ or aCT1/GSK/TMZ. These drug combinations did not increase TMZ alone-induced growth inhibition in astrocytes (Supplemental Fig. S14), suggesting that addition of aCT1 and p110 $\beta$-selective inhibitors does not exacerbate non-selective toxicity of TMZ to the normal brain. Collectively, our results demonstrate that simultaneously targeting $\mathrm{Cx} 43$ and $\mathrm{p} 110 \beta$ diminishes $T M Z$ resistance.

\section{DISCUSSION}

In this report, we have identified the molecular details underlying Cx43-induced MGMT-independent TMZ resistance. As illustrated in a model proposed in Fig. 6, Cx43 perhaps binds to p110 $\beta /$ p85 signaling complex upon receiving signals from extracellular cues (i.e., growth factors). This selective binding brings the $\mathrm{p} 110 \mathrm{\beta} /$ p85 signaling complex to the membrane and subsequently activates AKT. Activated PI3K/AKT signaling renders GBM cells resistant to $T M Z$, which is independent of MGMT. This model not only explains how a gap junction protein regulates chemoresistance through its non-channel functions but also provides a strong rationale for developing combinational therapies to overcome TMZ resistance. Indeed, aCT1 was found in the Cx43/p110 $\beta$ precipitates (Fig. 40), suggesting that this $C \times 43$-mimetic peptide likely blocks interactions between $\mathrm{Cx} 43$ and $\mathrm{p} 110 \beta$. It is therefore anticipated that $\mathrm{aCT} 1$ (blocking protein-protein interactions) and p110ß-selective inhibitors (blocking PI3K kinase activity) synergistically overcome TMZ resistance, which has been verified in vitro and in vivo in this report (Fig. 5).

Prior studies report that $\mathrm{C} \times 43 \mathrm{mRNA}$ and protein are detected in $\sim 20-60 \%$ of GBM patients $[15,41,47,48]$. In light of the fact that $45 \%$ of GBM patients express no MGMT $[6,7]$, there should be $10 \%$ $(20 \% \times 50 \%)$ to $30 \%(60 \% \times 50 \%)$ of patients that are MGMTdeficient and express high levels of Cx43. Congruent with this expectation, we have found that $16.7 \%$ of MGMT-deficient GBM patients express high levels of $\mathrm{Cx} 43$ [21]. That being said, around $20 \%$ of Cx43-high GBM patients may be refractory to TMZ treatment in the clinic. Therefore, the combinational treatment developed herein will benefit these patients, thereby having an important impact on future therapeutic intervention. Previous work has also revealed that, with the exception of Cx43, overexpression or inhibition of $\mathrm{C} \times 30, \mathrm{C} \times 32, \mathrm{C} \times 26$, or $\mathrm{C} \times 46$ also blocks the growth of rat or human glioma cells [63-70]. However, contradictory to these results, other studies show that $\mathrm{Cx} 30$ and

\section{MGMT-deficient and Cx43/p110 $\beta^{\text {high }}$ GBM}

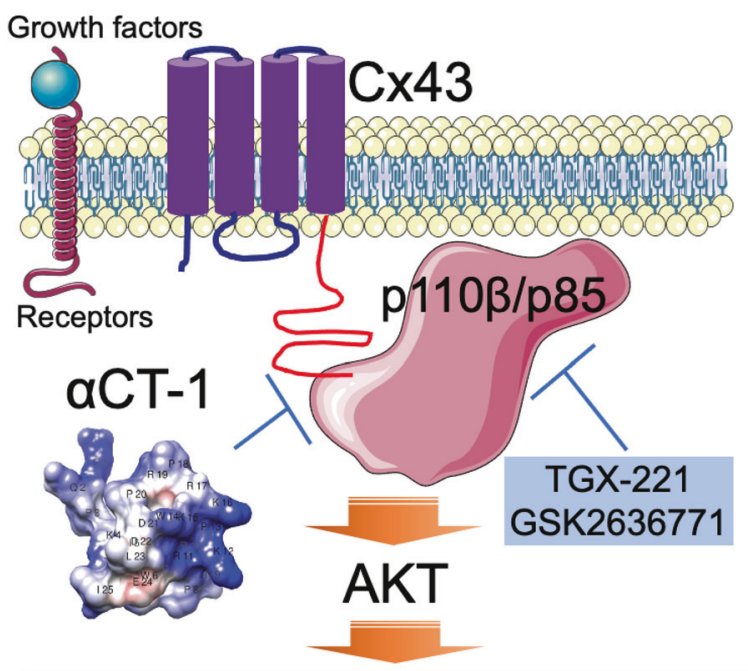

MGMT-independent TMZ resistance

Fig. 6 A model illustrates Cx43/PI3K-mediated TMZ resistance independent of MGMT. In MGMT-deficient GBMs, after growth factors bind to their cell surface receptors, $\mathrm{Cx} 43$ is expected to recruit $p 110 \beta / p 85$ signaling complexes to the membrane through a selective binding to $p 110 \beta$. This in turn activates PI3K/AKT signaling and induces MGMT-independent TMZ resistance. It is also expected that $\alpha \mathrm{CT} 1$, a mimetic peptide of $\mathrm{C} \times 43 \mathrm{CT}$, blocks the interaction between $\mathrm{C} x 43$ and $\mathrm{p} 110 \beta$, thereby inactivating $\mathrm{p} 110 \beta$ and overcoming TMZ resistance. Moreover, p110 $\beta$-selective inhibitors TGX221 and GSK2636771, which block p110 $\beta$ kinase activity, synergize with $\alpha C T 1$ to restore TMZ sensitivity in MGMT-deficient GBMs.

Cx32 have no effect on glioma growth [66, 71, 72]. In line with the fact that $\mathrm{Cx} 43$ levels are much higher than other connexins in GBM (Fig. 1) and the finding that $\mathrm{Cx} 43$ controls chemoresistance (Figs. 2-5), Cx43 is therefore the prime therapeutic target for GBM among all connexins.

Cx43 has long been considered as a tumor suppressor for glioma because overexpression of $\mathrm{Cx} 43$ leads to remarkable growth inhibition [73] and levels of Cx43 mRNA and protein inversely correlate with the aggressiveness of glioma [41]. However, drawbacks in these studies have made the tumorsuppressive activity of $\mathrm{Cx} 43$ questionable. For example, while 
ectopically expressing Cx43 does inhibit tumor cell growth, it is unclear whether the loss of endogenous $\mathrm{Cx} 43$ in normal glial cells promotes gliomagenesis as other tumor suppressors do, namely p53 and NF-1. Nonetheless, it is possible that gap junction intercellular communication controlled by $\mathrm{Cx} 43$ is GBM suppressive because the loss of this communication promotes oncogeneinduced transformation [74]. In contrast to these studies, we have established a tumor-promoting role of $\mathrm{Cx} 43$ in GBM. Cx43, whose mRNA levels are the highest among all connexins, correlates with GBM prognosis and chemoresistance. This membrane channel protein also activates $\mathrm{PI} 3 \mathrm{~K}$ independent of its channels, selectively binds to $\mathrm{p} 110 \beta$, and induces MGMT-independent TMZ resistance. Therefore, it is likely that $\mathrm{C} \times 43$ has multifaceted roles in GBM: Cx43-channels inhibit GBM formation, whereas the non-channel activities elicited by Cx43-CT (e.g., PI3K activation) confer chemoresistance during GBM progression.

\section{REFERENCES}

1. Tisdale MJ. Antitumor imidazotetrazines-XV. Role of guanine $\mathrm{O} 6$ alkylation in the mechanism of cytotoxicity of imidazotetrazinones. Biochem Pharmacol. 1987;36:457-62.

2. Portnow J, Badie B, Chen M, Liu A, Blanchard S, Synold TW. The neuropharmacokinetics of temozolomide in patients with resectable brain tumors: potential implications for the current approach to chemoradiation. Clin Cancer Res. 2009;15:7092-8.

3. Ostrom QT, Gittleman H, Truitt G, Boscia A, Kruchko C, Barnholtz-Sloan JS. CBTRUS statistical report: primary brain and other central nervous system tumors diagnosed in the United States in 2011-2015. Neuro Oncol. 2018;20:iv1-iv86.

4. Siegel RL, Miller KD, Jemal A. Cancer statistics, 2019. CA Cancer J Clin. 2019;69:7-34.

5. Weller M, Cloughesy T, Perry JR, Wick W. Standards of care for treatment of recurrent glioblastoma-are we there yet? Neuro Oncol. 2013;15:4-27.

6. Stupp R, Mason WP, van den Bent MJ, Weller M, Fisher B, Taphoorn MJ, et al. Radiotherapy plus concomitant and adjuvant temozolomide for glioblastoma. New Engl J Med. 2005;352:987-96.

7. Hegi ME, Diserens AC, Gorlia T, Hamou MF, de Tribolet N, Weller M, et al. MGMT gene silencing and benefit from temozolomide in glioblastoma. New Engl J Med. 2005;352:997-1003.

8. Quinn JA, Jiang SX, Reardon DA, Desjardins A, Vredenburgh JJ, Rich JN, et al. Phase II trial of temozolomide plus 06-benzylguanine in adults with recurrent, temozolomide-resistant malignant glioma. J Clin Oncol. 2009;27:1262-7.

9. Bocangel DB, Finkelstein S, Schold SC, Bhakat KK, Mitra S, Kokkinakis DM. Multifaceted resistance of gliomas to temozolomide. Clin Cancer Res. 2002;8:2725-34.

10. Happold C, Roth P, Wick W, Schmidt N, Florea AM, Silginer M, et al. Distinct molecular mechanisms of acquired resistance to temozolomide in glioblastoma cells. J Neurochem. 2012;122:444-55.

11. Cahill DP, Levine KK, Betensky RA, Codd PJ, Romany CA, Reavie LB, et al. Loss of the mismatch repair protein MSH6 in human glioblastomas is associated with tumor progression during temozolomide treatment. Clinical cancer research: an official journal of the American Association for. Cancer Res. 2007;13:2038-45.

12. Messaoudi $K$, Clavreul A, Lagarce F. Toward an effective strategy in glioblastoma treatment. Part I: resistance mechanisms and strategies to overcome resistance of glioblastoma to temozolomide. Drug Discov Today. 2015;20:899-905.

13. Palatinus JA, Rhett JM, Gourdie RG. The connexin43 carboxyl terminus and cardiac gap junction organization. Biochim Biophys Acta. 2012;1818:1831-43.

14. Chen W, Wang D, Du X, He Y, Chen S, Shao Q, et al. Glioma cells escaped from cytotoxicity of temozolomide and vincristine by communicating with human astrocytes. Med Oncol. 2015;32:43.

15. Gielen PR, Aftab Q, Ma N, Chen VC, Hong X, Lozinsky S, et al. Connexin43 confers Temozolomide resistance in human glioma cells by modulating the mitochondrial apoptosis pathway. Neuropharmacology 2013;75:539-48.

16. Munoz JL, Rodriguez-Cruz V, Greco SJ, Ramkissoon SH, Ligon KL, Rameshwar P. Temozolomide resistance in glioblastoma cells occurs partly through epidermal growth factor receptor-mediated induction of connexin 43. Cell Death Dis. 2014;5:e1145.

17. Lai SW, Huang BR, Liu YS, Lin HY, Chen CC, Tsai CF, et al. Differential characterization of temozolomide-resistant human glioma cells. Int J Mol Sci. 2018;19:127.

18. Yusubalieva GM, Baklaushev VP, Gurina OI, Zorkina YA, Gubskii IL, Kobyakov GL, et al. Treatment of poorly differentiated glioma using a combination of monoclonal antibodies to extracellular connexin-43 fragment, temozolomide, and radiotherapy. Bull Exp Biol Med. 2014;157:510-5.
19. Zhang XH, Qian Y, Li Z, Zhang NN, Xie YJ. Let-7g-5p inhibits epithelialmesenchymal transition consistent with reduction of glioma stem cell phenotypes by targeting VSIG4 in glioblastoma. Oncol Rep. 2016;36:2967-75.

20. Wang L, Peng Y, Peng J, Shao M, Ma L, Zhu Z, et al. Tramadol attenuates the sensitivity of glioblastoma to temozolomide through the suppression of Cx43mediated gap junction intercellular communication. Int J Oncol. 2018;52:295-304.

21. Murphy SF, Varghese RT, Lamouille S, Guo S, Pridham KJ, Kanabur P, et al. Connexin 43 inhibition sensitizes chemoresistant glioblastoma cells to temozolomide. Cancer Res. 2016;76:139-49.

22. Hunter AW, Barker RJ, Zhu C, Gourdie RG. Zonula occludens-1 alters connexin43 gap junction size and organization by influencing channel accretion. Mol Biol Cell. 2005;16:5686-98.

23. Kanabur P, Guo S, Simonds GR, Kelly DF, Gourdie RG, Verbridge SS, et al. Patientderived glioblastoma stem cells respond differentially to targeted therapies. Oncotarget 2016;7:86406-19.

24. Li T, Murphy S, Kiselev B, Bakshi KS, Zhang J, Eltahir A, et al. A new interleukin-13 amino-coated gadolinium metallofullerene nanoparticle for targeted mri detection of glioblastoma tumor cells. J Am Chem Soc. 2015;137:7881-8.

25. Pohlmann ES, Patel K, Guo S, Dukes MJ, Sheng Z, Kelly DF. Real-time visualization of nanoparticles interacting with glioblastoma stem cells. Nano Lett. 2015;15:2329-35.

26. Varghese RT, Liang Y, Guan T, Franck CT, Kelly DF, Sheng Z. Survival kinase genes present prognostic significance in glioblastoma. Oncotarget 2016;7:20140-51.

27. Pridham KJ, Le L, Guo S, Varghese RT, Algino S, Liang Y, et al. PIK3CB/p110beta is a selective survival factor for glioblastoma. Neuro Oncol. 2018;20:494-505.

28. Sheng KL, Pridham KJ, Sheng Z, Lamouille S, Varghese RT. Functional blockade of small GTPase ran inhibits glioblastoma cell viability. Front Oncol. 2018;8:662.

29. Varghese RT, Young S, Pham L, Liang Y, Pridham KJ, Guo S, et al. Casein kinase 1 epsilon regulates glioblastoma cell survival. Sci Rep. 2018;8:13621.

30. Roberts R, Smyth JW, Will J, Roberts P, Grek CL, Ghatnekar GS, et al. Development of PLGA nanoparticles for sustained release of a connexin43 mimetic peptide to target glioblastoma cells. Mater Sci Eng C Mater Biol Appl. 2020;108:110191.

31. Sheng Z, Li L, Zhu LJ, Smith TW, Demers A, Ross AH, et al. A genome-wide RNA interference screen reveals an essential CREB3L2-ATF5-MCL1 survival pathway in malignant glioma with therapeutic implications. Nat Med. 2010;16:671-7.

32. Sheng Z, Ma L, Sun JE, Zhu LJ, Green MR. BCR-ABL suppresses autophagy through ATF5-mediated regulation of mTOR transcription. Blood 2011;118:2840-8.

33. Den RB, Kamrava M, Sheng Z, Werner-Wasik M, Dougherty $E$, Marinucchi $M$, et al. A phase I study of the combination of sorafenib with temozolomide and radiation therapy for the treatment of primary and recurrent high-grade gliomas. Int J Radiat Oncol Biol Phys. 2013;85:321-8.

34. Ma L, Shan Y, Bai R, Xue L, Eide CA, Ou J, et al. A therapeutically targetable

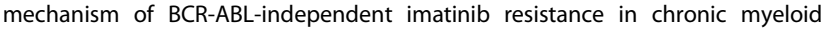
leukemia. Sci Transl Med. 2014;6:252ra121.

35. Guo S, Pridham KJ, Virbasius CM, He B, Zhang L, Varmark H, et al. A large-scale RNA interference screen identifies genes that regulate autophagy at different stages. Sci Rep. 2018;8:2822.

36. Sheng Z, Lewis JA, Chirico WJ. Nuclear and nucleolar localization of 18-kDa fibroblast growth factor- 2 is controlled by C-terminal signals. J Biol Chem. 2004;279:40153-60.

37. Sheng Z, Liang Y, Lin CY, Comai L, Chirico WJ. Direct regulation of rRNA transcription by fibroblast growth factor 2. Mol Cell Biol. 2005;25:9419-26.

38. Guo S, Liang Y, Murphy SF, Huang A, Shen H, Kelly DF, et al. A rapid and high content assay that measures cyto-ID-stained autophagic compartments and estimates autophagy flux with potential clinical applications. Autophagy 2015;11:560-72.

39. Wu S, Li X, Gao F, de Groot JF, Koul D, Yung WKA. PARP mediated PARylation of MGMT is critical to promote repair of temozolomide-induced O6-methylguanine DNA damage in glioblastoma. Neuro Oncol. 2021;23:920-31.

40. Liu Q, Yin X, Languino LR, Altieri DC. Evaluation of drug combination effect using a Bliss independence dose-response surface model. Stat Biopharm Res. 2018;10:112-22.

41. Grek CL, Sheng Z, Naus CC, Sin WC, Gourdie RG, Ghatnekar GG. Novel approach to temozolomide resistance in malignant glioma: connexin43-directed therapeutics. Curr Opin Pharmacol. 2018;41:79-88.

42. Caltabiano R, Torrisi A, Condorelli D, Albanese V, Lanzafame S. High levels of connexin 43 mRNA in high grade astrocytomas. Study of 32 cases with in situ hybridization. Acta Histochem. 2010;112:529-35.

43. Sin WC, Crespin S, Mesnil M. Opposing roles of connexin43 in glioma progression. Biochim Biophys Acta. 2012;1818:2058-67.

44. Huang RP, Hossain MZ, Sehgal A, Boynton AL. Reduced connexin43 expression in high-grade human brain glioma cells. J Surg Oncol. 1999;70:21-4.

45. Soroceanu L, Manning TJ Jr, Sontheimer H. Reduced expression of connexin-43 and functional gap junction coupling in human gliomas. Glia 2001;33:107-17. 
46. Pu P, Xia Z, Yu S, Huang Q. Altered expression of Cx43 in astrocytic tumors. Clin Neurol Neurosurg. 2004;107:49-54.

47. Crespin S, Fromont G, Wager M, Levillain P, Cronier L, Monvoisin A, et al. Expression of a gap junction protein, connexin43, in a large panel of human gliomas: new insights. Cancer Med. 2016;5:1742-52.

48. Cottin S, Gould PV, Cantin L, Caruso M. Gap junctions in human glioblastomas: implications for suicide gene therapy. Cancer Gene Ther. 2011;18:674-81.

49. Bowman RL, Wang Q, Carro A, Verhaak RG, Squatrito M. GlioVis data portal for visualization and analysis of brain tumor expression datasets. Neuro Oncol. 2017;19:139-41.

50. Zhao Z, Zhang KN, Wang Q, Li G, Zeng F, Zhang Y, et al. Chinese Glioma Genome Atlas (CGGA): a comprehensive resource with functional genomic data from chinese glioma patients. Genom. Proteom Bioinform. 2021;19:1-12.

51. Ghandi M, Huang FW, Jane-Valbuena J, Kryukov GV, Lo CC, McDonald ER 3rd, et al. Next-generation characterization of the Cancer Cell Line Encyclopedia. Nature 2019;569:503-8.

52. Uhlen $M$, Fagerberg $L$, Hallstrom BM, Lindskog $C$, Oksvold $P$, Mardinoglu $A$, et al. Proteomics. Tissue-based map of the human proteome. Science. 2015;347:1260419.

53. Larjavaara S, Mantyla R, Salminen T, Haapasalo H, Raitanen J, Jaaskelainen J, et al. Incidence of gliomas by anatomic location. Neuro Oncol. 2007;9:319-25.

54. Ek-Vitorin JF, King TJ, Heyman NS, Lampe PD, Burt JM. Selectivity of connexin 43 channels is regulated through protein kinase C-dependent phosphorylation. Circ Res. 2006;98:1498-505.

55. Li W, Hertzberg EL, Spray DC. Regulation of connexin43-protein binding in astrocytes in response to chemical ischemia/hypoxia. J Biol Chem. 2005;280:7941-8.

56. Leithe E, Mesnil M, Aasen T. The connexin 43 C-terminus: a tail of many tales. Biochim Biophys Acta. 2018;1860:48-64.

57. O'Quinn MP, Palatinus JA, Harris BS, Hewett KW, Gourdie RG. A peptide mimetic of the connexin43 carboxyl terminus reduces gap junction remodeling and induced arrhythmia following ventricular injury. Circ Res. 2011;108:704-15.

58. Chi Y, Gao K, Li K, Nakajima S, Kira S, Takeda M, et al. Purinergic control of AMPK activation by ATP released through connexin 43 hemichannels - pivotal roles in hemichannel-mediated cell injury. J Cell Sci. 2014;127:1487-99.

59. Ujiie H, Chaytor AT, Bakker LM, Griffith TM. Essential role of Gap junctions in NOand prostanoid-independent relaxations evoked by acetylcholine in rabbit intracerebral arteries. Stroke 2003;34:544-50.

60. Fiori MC, Reuss L, Cuello LG, Altenberg GA. Functional analysis and regulation of purified connexin hemichannels. Front Physiol. 2014;5:71.

61. Pridham KJ, Varghese RT, Sheng Z. The role of class IA phosphatidylinositol-4,5bisphosphate 3-kinase catalytic subunits in glioblastoma. Front Oncol. 2017;7:312.

62. Mateo J, Ganji G, Lemech C, Burris HA, Han SW, Swales KE, et al. A first-time-inhuman study of GSK2636771, a phosphoinositide 3 kinase beta-selective inhibitor, in patients with advanced solid tumors. Clin Cancer Res. 2017;23:5981-92.

63. Mulkearns-Hubert EE, Torre-Healy LA, Silver DJ, Eurich JT, Bayik D, Serbinowski E, et al. Development of a Cx46 targeting strategy for cancer stem cells. Cell Rep. 2019;27:1062-72.e5.

64. Hitomi M, Deleyrolle LP, Mulkearns-Hubert EE, Jarrar A, Li M, Sinyuk $M$, et al. Differential connexin function enhances self-renewal in glioblastoma. Cell Rep. 2015;11:1031-42

65. Jimenez T, Fox WP, Naus CC, Galipeau J, Belliveau DJ. Connexin over-expression differentially suppresses glioma growth and contributes to the bystander effect following HSV-thymidine kinase gene therapy. Cell Commun Adhes. 2006;13:79-92.

66. Goldberg GS, Bechberger JF, Tajima Y, Merritt M, Omori Y, Gawinowicz MA, et al. Connexin43 suppresses MFG-E8 while inducing contact growth inhibition of glioma cells. Cancer Res. 2000;60:6018-26.

67. Yoshimura T, Satake M, Ohnishi A, Tsutsumi Y, Fujikura Y. Mutations of connexin32 in Charcot-Marie-Tooth disease type $X$ interfere with cell-to-cell communication but not cell proliferation and myelin-specific gene expression. J Neurosci Res. 1998;51:154-61.

68. Arun S, Ravisankar S, Vanisree AJ. Implication of connexin30 on the stemness of glioma: connexin 30 reverses the malignant phenotype of glioma by modulating IGF-1R, CD133 and CMyc. J Neurooncol. 2017;135:473-85.

69. Arun S, Vanisree AJ, Ravisankar S. Connexin 30 downregulates Insulin-like growth factor receptor-1, abolishes Erk and potentiates effects of an IGF-R inhibitor in a glioma cell line. Brain Res. 2016;1643:80-90.

70. Artesi M, Kroonen J, Bredel M, Nguyen-Khac M, Deprez M, Schoysman L, et al. Connexin 30 expression inhibits growth of human malignant gliomas but protects them against radiation therapy. Neuro Oncol. 2015;17:392-406.

71. Cotrina ML, Lin JH, Nedergaard M. Adhesive properties of connexin hemichannels. Glia 2008;56:1791-8.
72. Fu CT, Bechberger JF, Ozog MA, Perbal B, Naus CC. CCN3 (NOV) interacts with connexin43 in C6 glioma cells: possible mechanism of connexin-mediated growth suppression. J Biol Chem. 2004;279:36943-50.

73. Naus CC, Elisevich K, Zhu D, Belliveau DJ, Del Maestro RF. In vivo growth of C6 glioma cells transfected with connexin43 cDNA. Cancer Res. 1992;52:4208-13.

74. Aasen T, Mesnil M, Naus CC, Lampe PD, Laird DW. Gap junctions and cancer: communicating for 50 years. Nat Rev Cancer. 2016;16:775-88.

\section{ACKNOWLEDGEMENTS}

This study is supported by National Institutes of Health (NIH) R21 grants R21CA216768 and R21CA245631 to ZS, NIH R01 grants HL56728 and HL141855 to RGG, St Baldrick's Foundation Summer Medical Student Fellowships to FS and PK, Summer Research Fellowship from Virginia Tech Carilion School of Medicine to $\mathrm{KH}_{\text {, }}$ and Translational Neurobiology Summer Undergraduate Research Fellowships from the Fralin Biomedical Research Institute to MM and GL. The results shown in Fig. 1 are in part based upon data generated by the TCGA Research Network: https://www. cancer.gov/tcga.

\section{AUTHOR CONTRIBUTIONS}

Conception and design: ZS, KJP, and RGG. Development of methodology: KJP, FS, KH $S G, S L, J J, R V$, and ZS. Acquisition of data (performed experiments, provided reagents, etc.): KJP, FS, KH, KLS, SG, ML, PK, SL, GL, MM, JJ, RV, and DFK. Analysis and interpretation of data (e.g., statistical analysis, computational analysis): KJP, KH, KLS, RV, and ZS. Assistance in data interpretation: CLG and GGG. Writing, review, and/or revision of the manuscript: $Z S, R G G$, and KJP.

\section{COMPETING INTERESTS}

GGG is CEO, President and co-founder of FirstString Research Inc, which licensed aCT1 peptide. CLG is Senior Director of Research and Development at FirstString Research Inc. RGG is a non-remunerated member of the Scientific Advisory Board of FirstString Research, as well as a co-founder of the company. GGG, RGG, JJ, and CLG have ownership interests in FirstString Research Inc. The remaining authors have no disclosures to report.

\section{ADDITIONAL INFORMATION}

Supplementary information The online version contains supplementary material available at https://doi.org/10.1038/s41389-022-00378-7.

Correspondence and requests for materials should be addressed to Robert G. Gourdie or Zhi Sheng.

Reprints and permission information is available at http://www.nature.com/ reprints

Publisher's note Springer Nature remains neutral with regard to jurisdictional claims in published maps and institutional affiliations.

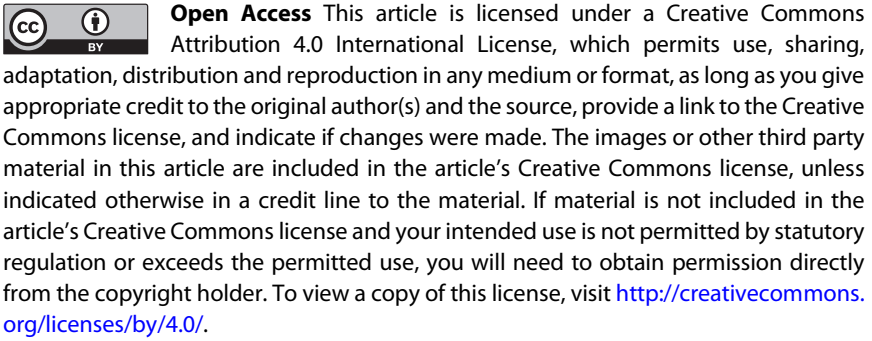

(c) The Author(s) 2022 